\title{
Schiffer Comparison Operators and Approximations on Riemann Surfaces Bordered by Quasicircles
}

\author{
Eric Schippers ${ }^{1} \cdot$ Mohammad Shirazi $^{1} \cdot$ Wolfgang Staubach $^{2}$ \\ Received: 15 March 2020 / Accepted: 2 September 2020 / Published online: 24 September 2020 \\ (c) The Author(s) 2020
}

\begin{abstract}
We consider a compact Riemann surface $R$ of arbitrary genus, with a finite number of non-overlapping quasicircles, which separate $R$ into two subsets: a connected Riemann surface $\Sigma$, and the union $\mathcal{O}$ of a finite collection of simply connected regions. We prove that the Schiffer integral operator mapping the Bergman space of anti-holomorphic one-forms on $\mathcal{O}$ to the Bergman space of holomorphic forms on $\Sigma$ is an isomorphism onto the exact one-forms, when restricted to the orthogonal complement of the set of forms on all of $R$. We then apply this to prove versions of the Plemelj-Sokhotski isomorphism and jump decomposition for such a configuration. Finally we obtain some approximation theorems for the Bergman space of one-forms and Dirichlet space of holomorphic functions on $\Sigma$ by elements of Bergman space and Dirichlet space on fixed regions in $R$ containing $\Sigma$.
\end{abstract}

Keywords Approximation · Riemann surfaces · Quasicircles · Schiffer operators

Mathematics Subject Classification Primary: 58C99 - 58A10 - 30F10 - 30F15 . 30F30 $\cdot 32 \mathrm{~W} 05 \cdot$ Secondary: 58J40

$\bowtie \quad$ Wolfgang Staubach

wulf@math.uu.se

Eric Schippers

eric.schippers@umanitoba.ca

Mohammad Shirazi

mohammad.shirazi@mail.mcgill.ca

1 Machray Hall, Department of Mathematics, University of Manitoba, Winnipeg, MB R3T 2N2,

Canada

2 Department of Mathematics, Uppsala University, 75106 Uppsala, Sweden 


\section{Introduction and Statement of the Main Theorems}

\subsection{Introduction}

In earlier publications [18-20], two of the authors developed a theory of the transmission of harmonic Dirichlet-bounded functions across curves, and of the related Plemelj-Sokhotski jump formula on such curves. The curves were assumed to be quasicircles, which are not rectifiable in general. This theory involves certain singular integral operators due to M. Schiffer, which are also intimately related to approximations of holomorphic functions and forms, through the Faber and Grunsky operators and their generalizations. A number of results strongly indicate that quasicircles are the natural curves for this circle of ideas.

In this paper, we apply these techniques to derive approximation theorems for nested multiply connected domains in Riemann surfaces of arbitrary genus. We show that, given a compact Riemann surface $R$ with nested regions $\Sigma \subseteq \Sigma^{\prime} \subset R$ obtained by removing disks from $R$, under certain general conditions, the Dirichlet spaces of functions and Bergman spaces of one-forms on $\Sigma^{\prime}$ are dense in the Dirichlet spaces and Bergman spaces of $\Sigma$, respectively. To do so, we must first extend two of our results from the case of one curve separating the surface to many curves bounding conformal disks. Namely, we must extend the Schiffer isomorphism to this case, as well as the Plemelj-Sokhotski jump isomorphism and decomposition. These results are perhaps interesting on their own, and have several applications (see below). Precise statements of the theorems, as well as discussion of related literature, are given in Sect. 1.3, after the necessary preliminaries are dealt with in Sect. 1.2. The remainder of the paper is devoted to the proofs.

It should be observed that there are very few results involving approximation in the $L^{2}$ norm by functions/forms on a larger region. The literature has so far been largely concerned with uniform approximation, e.g., Gauthier and Sharifi [7]; for literature reviews see F. Sharifi [21] and Fornæss et al. [5]. To our knowledge the only result of this form is that of Askaripour and Barron [2].

This paper is part of an ongoing investigation of Faber, Grunsky, and Schiffer operators on Riemann surfaces split in pieces by quasicircles. This has applications to Teichmüller theory [11], two-dimensional conformal field theory [13], and approximation theory [12] (as in the present paper).

\subsection{Preliminaries}

Let $\Sigma$ be a Riemann surface. Define the pairing of one-forms on $\Sigma$

$$
\left(\omega_{1}, \omega_{2}\right)=\frac{1}{2} \iint_{\Sigma} \omega_{1} \wedge * \overline{\omega_{2}}
$$

Let $A(\Sigma)$ denote the set of holomorphic one-forms on $\Sigma$ for which this pairing is finite. Similarly, let $\overline{A(\Sigma)}$ denote the set of anti-holomorphic one-forms for which (1.1) is finite. Denote the set of harmonic one-forms such that this pairing is finite by $A_{\text {harm }}(\Sigma)$. We have the orthogonal decomposition 


$$
A_{\text {harm }}(\Sigma)=A(\Sigma) \oplus \overline{A(\Sigma)}
$$

The subscript $e$ will denote the subset of exact forms; e.g., $A(\Sigma)_{e}, A_{\text {harm }}(\Sigma)_{e}$ etc.

We also define the Dirichlet spaces. Let

$$
\begin{aligned}
\mathcal{D}_{\text {harm }}(\Sigma) & =\left\{h: \Sigma \rightarrow \mathbb{C} \text { harmonic }: d h \in A_{\text {harm }}(\Sigma)\right\} \\
\mathcal{D}(\Sigma) & =\{h: \Sigma \rightarrow \mathbb{C} \text { holomorphic }: \partial h \in A(\Sigma)\} \\
\overline{\mathcal{D}(\Sigma)} & =\{h: \Sigma \rightarrow \mathbb{C} \text { anti-holomorphic }: \bar{\partial} h \in \overline{A(\Sigma)}\} .
\end{aligned}
$$

For a point $q \in \Sigma$, the subscript $q$ will denote the subset of functions vanishing at $q$; e.g., $\mathcal{D}_{\text {harm }}(\Sigma)_{q}$, etc.

Denote complex conjugation of functions $h$ and forms $\alpha$ by $\bar{h}$ and $\bar{\alpha}$. Of course, $\overline{\mathcal{D}(\Sigma)}$ consists of the set of complex conjugates of elements of $\mathcal{D}(\Sigma)$, justifying the notation. The notation $\overline{A(\Sigma)}$ is similarly justified.

By a conformal map, we mean a holomorphic map which is a biholomorphism onto its image. To define a quasiconformal map we first need the notion of Beltrami differential on $\Sigma$, which is a $(-1,1)$-differential $\omega$ on $\Sigma$, i.e., a differential given in a local biholomorphic coordinate $z$ by $\mu(z) d \bar{z} / d z$, such that $\mu$ is Lebesgue-measurable in every choice of coordinate and $\|\mu\|_{\infty}<1$. Quasiconformal maps are by definition solutions to the Beltrami equation, i.e., the differential equation given in local coordinates by $\bar{\partial} f=\omega \partial f$ where $\omega$ is a Beltrami differential on $\Sigma$. Let $\mathbb{C}$ denote the complex plane and $\overline{\mathbb{C}}$ denote the Riemann sphere. By a quasicircle in the plane, we mean the image of the unit circle $\mathbb{S}^{1}=\{z \in \mathbb{C}:|z|=1\}$ under a quasiconformal mapping of the plane. By a quasicircle $\Gamma$ in $\Sigma$, we mean a simple closed curve such that there is a conformal map $\phi: U \rightarrow \mathbb{C}$ such that $U$ is an open neighborhood of $\Gamma$ and $\phi(\Gamma)$ is a quasicircle in $\mathbb{C}$ in the sense above.

Let $R$ be a compact Riemann surface. Fix points $z, q$, and $w_{0} \in R$. Following, for example, H. Royden [14], we define Green's function of $R$ to be the unique function $\mathscr{G}\left(w, w_{0} ; z, q\right)$ such that

(1) $\mathscr{G}$ is harmonic in $w$ on $R \backslash\{z, q\}$;

(2) for a local coordinate $\phi$ on an open set $U$ containing $z, \mathscr{G}\left(w, w_{0} ; z, q\right)+$ $\log |\phi(w)-\phi(z)|$ is harmonic for $w \in U$;

(3) for a local coordinate $\phi$ on an open set $U$ containing $q, \mathscr{G}\left(w, w_{0} ; z, q\right)-$ $\log |\phi(w)-\phi(q)|$ is harmonic for $w \in U$;

(4) $\mathscr{G}\left(w_{0}, w_{0} ; z, q\right)=0$ for all $z, q, w_{0}$.

It can be shown that $g$ exists, and is uniquely determined by these properties. The normalization at $w_{0}$ is inconsequential, because it can be shown that $\partial_{w} \mathscr{G}\left(w, w_{0} ; z, q\right)$ and $\bar{\partial}_{w} \mathscr{G}\left(w, w_{0} ; z, q\right)$ are independent of $w_{0}$. Thus we leave $w_{0}$ out of the notation for $\mathscr{G}$. Also, $\mathscr{G}$ is harmonic in both $w$ and $z$.

Let $R$ be a compact Riemann surface as above, and $\Sigma \subset R$ be an open, proper, and connected subset which we treat as a Riemann surface. For such surfaces we have a different notion of Green's function. We say that $\Sigma$ has a Green's function if there is a harmonic function $\mathscr{G}(z, w)$ on $\Sigma$ such that

(1) for a local coordinate $\phi$ on an open set $U \subset \Sigma$ containing $w, \mathscr{G}(z ; w)+\log \mid \phi(z)-$ $\phi(w) \mid$ is harmonic in $z$ on $U$; 
(2) $\lim _{z \rightarrow p} \mathscr{G}(z, w)=0$ for all $p \in \partial \Sigma$ and $w \in \Sigma$.

It can be shown that $\mathscr{G}$ is also harmonic in $w$.

It is always understood that we use the first type of Green's function for compact surfaces, and the second type for open proper connected subsets. When necessary, we distinguish between Green's functions of different surfaces with a subscript, e.g., $\mathscr{G}_{R}$ or $\mathscr{G}_{\Sigma}$.

\subsection{Statement of Results}

Let $R$ be a compact surface and $\mathcal{O}$ be an open subset. In this paper, we will always assume that $\mathcal{O}=\Omega_{1} \cup \ldots \cup \Omega_{n}$ where $\Omega_{k}$ are simply connected domains for $k=$ $1, \ldots, n$, each bounded by a quasicircle, whose closures are pairwise disjoint. Let $\Sigma$ be the complement of the closure of $\mathcal{O}$ in $R$.

We define the integral operators

$$
\begin{aligned}
T(\mathcal{O}, \Sigma): \overline{A(\mathcal{O})} & \rightarrow A(\Sigma) \\
\bar{\alpha} & \mapsto \frac{1}{\pi i} \iint_{\mathcal{O}} \partial_{z} \partial_{w} \mathscr{G}_{R}(w ; z, q) \wedge_{w} \overline{\alpha(w)}
\end{aligned}
$$

where $z \in \Sigma$. We refer to the kernel of this integral operator $L_{R}(z, w)=$ $\partial_{z} \partial_{w} \mathscr{G}_{R}(w ; z, q) /(\pi i)$ as the Schiffer kernel. Note that the above case includes the possibility that the domain $\mathcal{O}$ is connected; we will frequently use this case, for example, when restricting to one of the connected components $\Omega_{k}$ of $\mathcal{O}$. In that case we will denote the complement of the closure of $\Omega_{k}$ by $\Omega_{k}^{*}$. The fact that $T(\mathcal{O}, \Sigma)$ is bounded and has codomain $A(\Sigma)$ will be justified ahead.

Let

$$
V=\left\{\bar{\alpha} \in \overline{A(\mathcal{O})}: \iint_{\mathcal{O}} \beta \wedge \bar{\alpha}=0 \forall \beta \in A(R)\right\} .
$$

that is, $V$ is the orthogonal complement of $\left.\overline{A(R)}\right|_{\mathcal{O}}$ in $\overline{A(\mathcal{O})}$. The case that $R$ is of genus zero is qualitatively distinct. In this case, $A(R)=\{0\}$, and thus $V=\overline{A(\mathcal{O})}$.

Our first result is the following:

Theorem 1.1 Let $R$ be a compact Riemann surface, and let $\mathcal{O}=\Omega_{1} \cup \cdots \cup \Omega_{n}$ where $\Omega_{k}$ are simply connected domains in $R$ bounded by quasicircles $\Gamma_{k}$ for $k=1, \ldots, n$. Assume that the closures of $\Omega_{k}$ are pairwise disjoint. Let $\Sigma$ be the complement of the closure of $\mathcal{O}$ in $R$. Then the restriction of $T(\mathcal{O}, \Sigma)$ to $V$ is a bounded isomorphism onto $A(\Sigma)_{e}$.

This generalizes one direction of a result of V.V. Napalkov and R.S. Yulmukhametov [8], which says that in the case that $n=1$ and $R=\overline{\mathbb{C}}$, the Schiffer operator is an isomorphism if and only if the domain $\Omega$ is bounded by a quasicircle. This is closely related to a result proven by Schippers and Staubach [18] which shows that the jump decomposition in the plane results in a bounded isomorphism if and only if the curve 
is a quasicircle, and also a result of Y. Shen [22] which shows that the boundary of $\Omega$ is a quasicircle if and only if a certain sequential Faber operator is a bounded isomorphism. These results motivate the particular interest in Schiffer operators for regions bounded by quasicircles. Schippers and Staubach proved this theorem [20] in the case of a single quasicircle dividing a compact Riemann surface into two disjoint connected components.

We also give three applications of Theorem 1.1. First, we prove a version of the Plemelj-Sokhotski jump formula for $\Gamma=\Gamma_{1} \cup \cdots \cup \Gamma_{n}$ where $\Gamma_{k}$ are as in Theorem 1.1. By $\mathcal{H}(\Gamma)$, we mean the set of complex functions on $\Gamma$ whose restriction to $\Gamma_{k}$ is the boundary values of an element of $\mathcal{D}_{\text {harm }}\left(\Omega_{k}\right)$ in a sense which we refer to as "conformally non-tangential (CNT)" (see Sect. 2.2 for the precise definition). Let

$$
W=\left\{g \in \mathcal{D}_{\text {harm }}(\mathcal{O}): \bar{\partial} g \in V\right\} .
$$

In the genus zero case, we have that $W=\mathcal{D}_{\text {harm }}(\mathcal{O})$.

Theorem 1.2 Let $R, \Gamma, \Omega_{k}$ and $\Sigma$ be as in Theorem 1.1, and let $H \in \mathcal{H}(\Gamma)$ be such that its extension $h$ to $\mathcal{D}_{\text {harm }}(\mathcal{O})$ is in $W$. Fix $q \in \Sigma$. There are unique $h_{k} \in \mathcal{D}\left(\Omega_{k}\right)$, $k=1, \ldots, n$, and $h_{\Sigma} \in \mathcal{D}(\Sigma)_{q}$ so that if $H_{k}, H_{\Sigma}$ are their CNT boundary values, then on each curve $\Gamma_{k}, H=-H_{\Sigma}+H_{k}$. These are given by

$$
h_{k}=\left.J_{q}(\Gamma) h\right|_{\Omega_{k}}
$$

for $k=1, \ldots, n$, and

$$
h_{\Sigma}=\left.J_{q}(\Gamma) h\right|_{\Sigma}
$$

Here, $J_{q}(\Gamma)$ is an integral operator similar to the Cauchy integral, with integral kernel $-\partial_{w} g(w ; z, q) /(\pi i)$. See Sect. 2.1 ahead for the precise definition, which involves approximations of the quasicircles by analytic curves.

It is classically known that there is such a jump decomposition for reasonably smooth curves and functions on Riemann surfaces; see $[6,15]$. This was generalized to the case of a single quasicircle separating a compact Riemann surface into two components, and data in $\mathcal{H}(\Gamma)$ as above, in [20]. A discussion of the literature can also be found there. The space $W$ corresponds to the classical condition for existence of a jump; see Sect. 3.1 ahead.

The second application is an approximation theorem for Dirichlet spaces of holomorphic functions and Bergman spaces of holomorphic one-forms.

Theorem 1.3 Let $R$ be a compact Riemann surface and let $\Sigma, \Omega_{k}, \Gamma_{k}$ and $\Sigma^{\prime}, \Omega_{k}^{\prime}, \Gamma_{k}^{\prime}$ each be as in Theorem 1.1. Assume further that $\Sigma \subset \Sigma^{\prime}$ and that the quasicircles $\Gamma_{k}^{\prime}$ are isotopic to $\Gamma_{k}$ within the closure of $\Omega_{k}^{\prime}$ for each $k=1, \ldots, n$.

If $\Sigma^{\prime \prime} \subset R$ is any open set such that $\Sigma \subseteq \Sigma^{\prime \prime} \subseteq \Sigma^{\prime}$, then

(1) the set of restrictions of elements of $\mathcal{D}\left(\Sigma^{\prime \prime}\right)$ to $\Sigma$ is dense in $\mathcal{D}(\Sigma)$

(2) the set of restrictions of $A\left(\Sigma^{\prime \prime}\right)$ is dense in $A(\Sigma)$. 
Since one may always view $\Sigma^{\prime}$ as embedded in its double, one can remove the mention of the outer surface and obtain

Corollary 1.4 Let $\Sigma^{\prime}$ be a bordered Riemann surface whose boundary consists of $n$ curves $\Gamma_{1}^{\prime}, \ldots, \Gamma_{n}^{\prime}$ homeomorphic to $\mathbb{S}^{1}$, whose double is compact. Assume that $\Sigma \subset$ $\Sigma^{\prime}$ is an open set bordered by $n$ quasicircles $\Gamma_{1}, \ldots, \Gamma_{n}$, such that $\Gamma_{k}$ is isotopic to $\Gamma_{k}^{\prime}$ in $\left(\Sigma^{\prime} \cup \partial \Sigma^{\prime}\right) \backslash \Sigma$ for $k=1, \ldots, n$. For any open set $\Sigma^{\prime \prime}$ such that $\Sigma \subseteq \Sigma^{\prime \prime} \subseteq \Sigma^{\prime}$,

(1) the set of restrictions of $\mathcal{D}\left(\Sigma^{\prime \prime}\right)$ to $\Sigma$ is dense in $\mathcal{D}(\Sigma)$.

(2) the set of restrictions of elements of $A\left(\Sigma^{\prime \prime}\right)$ to $\Sigma$ is dense in $A(\Sigma)$.

Here, note that we mean that the boundaries are borders [1].

These results should be compared to a result of Askaripour and Barron [2], which says that if $D_{1}$ and $D_{2}$ are open subsets of a Riemann surface $R$ such that $D_{1} \subseteq D_{2}$, and the lift to the universal cover (the disk $\mathbb{D}$ ) of $D_{1}$ and $D_{2}$ are Carathéodory sets contained in a smaller disk, then restrictions of elements of the Bergman space $A\left(D_{2}\right)$ to $D_{1}$ are dense in $A\left(D_{1}\right)$. As mentioned in the introduction, as far as we know, their result is the first general result for nested Riemann surfaces for $L^{2}$ approximability, as opposed to uniform approximation. The approach of Askaripour and Barron uses a lift to the universal cover and application of Poincaré series. It would be of great interest to obtain our approximation theorems by applying their methods. Our approach here ultimately relies on sewing.

The third application involves another kind of operator which we now define. Let $R$ be a compact Riemann surface, $\Sigma$ and $\Sigma^{\prime}$ be Riemann surfaces such that $\Sigma \subset \Sigma^{\prime} \subset R$ and such that $\operatorname{cl} \Sigma \subset \Sigma^{\prime}$ (where the closure is with respect to the topology of $R$ ) and the inclusion maps from $\Sigma$ to $\Sigma^{\prime}$ and $\Sigma^{\prime}$ to $R$ are holomorphic.

$$
\begin{aligned}
S\left(\Sigma, \Sigma^{\prime}\right): A(\Sigma) & \rightarrow A\left(\Sigma^{\prime}\right) \\
\alpha & \mapsto-\frac{1}{\pi i} \iint_{\Sigma} \partial_{z} \bar{\partial}_{w} \mathscr{G}_{\Sigma^{\prime}}(w ; z, q) \wedge_{w} \alpha(w)
\end{aligned}
$$

The kernel of this integral operator

$$
K_{\Sigma^{\prime}}(z, w)=-\frac{1}{\pi i} \partial_{z} \bar{\partial}_{w^{\prime}} \mathscr{G}_{\Sigma^{\prime}}(w ; z, q)
$$

is the Bergman kernel of $\Sigma^{\prime}$. Note, however, that we integrate only over $\Sigma$ and not all of $\Sigma^{\prime}$.

We then have

Theorem 1.5 Let $R, \Sigma$, and $\Sigma^{\prime}$ be as in Theorem 1.3. Then $S\left(\Sigma, \Sigma^{\prime}\right)$ has trivial kernel and dense image.

The operators $T(\mathcal{O}, \Sigma)$ and $S\left(\Sigma, \Sigma^{\prime}\right)$ are special cases of what we call Schiffer comparison operators. Note that the domain of integration of $T(\mathcal{O}, \Sigma)$ is the subset $\mathcal{O}$ of $R$, and thus the operator depends on both $R$ and $\mathcal{O}$. Similarly, $S\left(\Sigma, \Sigma^{\prime}\right)$ depends on both $\Sigma^{\prime}$ and $\Sigma$. In general, we are interested in the extent to which information about 
the two surfaces $\mathcal{O}$ and $\Sigma$, or $\Sigma$ and $\Sigma^{\prime}$, is reflected in the properties of the Schiffer operators.

M. Schiffer and others $[3,4,16,17]$ have investigated these comparison operators in many cases. The Riemann surface $R$ might be the Riemann sphere, or a subset of the plane bounded by analytic curves; while the subset $\mathcal{O}$ might be a multiply connected planar domain or a subdomain of a compact surface $R$.

\section{The Jump and Schiffer Comparison Operators}

\subsection{A Cauchy-Type Operator on Compact Surfaces and a Schiffer Comparison Operator}

In this section we bring together various identities for the integral operators, and generalize some of them to the case of several boundary curves. These include expressions for the integral operators in terms of a kind of Cauchy-integral.

We begin with the case of one boundary curve. Let $R$ be a compact Riemann surface and let $\Gamma$ be a quasicircle, whose complement we assume to consist of two connected components $\Omega$ and $\Sigma$. Let $\mathscr{G}_{\Omega}$ denote the Green's function of $\Omega$, and for fixed $p \in \Omega$ and $s>0$ let $\Gamma_{s}^{p}$ be the level curves $\left\{w: \mathscr{G}_{\Omega}(w, p)=s\right\}$. For $s$ sufficiently small, these are in fact analytic simple closed curves, and we endow them with a positive orientation with respect to $p$. Fixing $q \in \Sigma$, we define

$$
\begin{aligned}
J_{q}(\Gamma): \mathcal{D}_{\text {harm }}(\Omega) & \rightarrow \mathcal{D}_{\text {harm }}(\Omega \cup \Sigma)_{q} \\
h & \mapsto-\lim _{s \searrow 0} \frac{1}{\pi i} \int_{\Gamma_{s}^{p}} \partial_{w} \mathscr{G}_{R}(w ; z, q) h(w)
\end{aligned}
$$

for $z \in R \backslash \Gamma$. This operator indeed takes $\mathcal{D}_{\text {harm }}(\Omega)$ into $\mathcal{D}_{\text {harm }}(\Omega \cup \Sigma)$ by [20, Corollary 4.3], where by the latter we mean a function on the disjoint union which is harmonic on $\Omega$ and $\Sigma$. The output vanishes at $q$ by [20, Theorem 4.26], and is a consequence of the fact that $\partial_{w} \mathscr{G}(w ; z, q)$ vanishes identically at $z=q$, which was established in the proof of that theorem. It was furthermore shown that the operator is bounded and independent of $p$. We may write the level curves $\Gamma_{s}^{p}$ in terms of a conformal map $f: \mathbb{D} \rightarrow \Omega$ such that $f(0)=p$, as the images $f\left(\left\{z:|z|=e^{-s}\right\}\right)$ of circles centered at 0 .

Recall the operator $T(\mathcal{O}, \Sigma)$ defined by (1.2) in the introduction. Specializing to the case that $\mathcal{O}$ consists of a single simply connected domain $\Omega$, yields an operator which we denote by $T(\Omega, \Sigma)$. It follows from [20, Theorem 3.9] that this operator is bounded. We also define

$$
\begin{aligned}
T(\Omega, \Omega): \overline{A(\Omega)} & \rightarrow A(\Omega) \\
\bar{\alpha} & \mapsto \frac{1}{\pi i} \iint_{\Omega} \partial_{z} \partial_{w} \mathscr{G}_{R}(w ; z, q) \wedge_{w} \overline{\alpha(w)}
\end{aligned}
$$

where $z \in \Omega$, which is also bounded by [20, Theorem 3.9]. 
Finally define

$$
\begin{aligned}
S(\Omega, R): A(\Omega) & \rightarrow A(R) \\
\alpha & \mapsto-\frac{1}{\pi i} \iint_{\Omega} \partial_{z} \bar{\partial}_{w} \mathscr{G}_{R}(w ; z, q) \wedge_{w} \alpha(w)
\end{aligned}
$$

for $z, q \in R$, which is bounded because the kernel function is globally bounded [20].

The conjugate operator is defined by

$$
\begin{aligned}
\bar{S}(\Omega, R): \overline{A(\Omega)} & \rightarrow \overline{A(R)} \\
\bar{\alpha} & \mapsto \overline{S(\Omega, R) \alpha .}
\end{aligned}
$$

Conjugates of $T$ operators are defined similarly.

If $R$ has genus zero, then it can be checked directly that $\partial_{z} \partial_{w} \mathscr{G}_{R}(w ; z, q)=0$. Thus the operators $S(\Omega, R)$ and $\bar{S}(\Omega, R)$ are both zero.

The operators $J_{q}(\Gamma), T(\Omega, \Sigma), T(\Omega, \Omega)$ and $S(\Omega, R)$ satisfy the identities [20, Theorem 4.2]

$$
\begin{array}{lrl}
\partial J_{q}(\Gamma) h(z)=-T(\Omega, \Sigma) \bar{\partial} h(z), & z \in \Sigma \\
\partial J_{q}(\Gamma) h(z)=\partial h(z)-T(\Omega, \Omega) \bar{\partial} h(z), & z \in \Omega \\
\bar{\partial} J_{q}(\Gamma) h(z)=\bar{S}(\Omega, R) \bar{\partial} h(z), & z \in \Omega \cup \Sigma .
\end{array}
$$

In the case that $R$ has genus zero, the right-hand side of the third equation is equal to zero by the observations above.

We would like to generalize these identities to the case of many boundary curves. First, we make a general remark on notation.

Remark 2.1 (Direct sum notation) Let $\mathcal{O}$ be as in the introduction; that is, $\mathcal{O}=$ $\Omega_{1} \cup \ldots \cup \Omega_{n}$ for $\Omega_{k}$ simply connected, bordered by quasicircles, with pairwise disjoint closures. In that case, we have a natural isomorphism

$$
\begin{aligned}
A(\mathcal{O}) & \stackrel{\cong}{\rightarrow} \bigoplus_{k=1}^{n} A\left(\Omega_{k}\right) \\
\alpha & \mapsto\left(\left.\alpha\right|_{\Omega_{1}}, \ldots,\left.\alpha\right|_{\Omega_{n}}\right) .
\end{aligned}
$$

The inverse of this isomorphism is

$$
\left(\alpha_{1}, \ldots, \alpha_{n}\right) \rightarrow \sum_{k=1}^{n} \alpha_{k} \chi_{k}
$$

where $\chi_{k}$ are the characteristic functions of $\Omega_{k}$ for $k=1, \ldots, n$. To avoid needless insertion of this isomorphism into every formula, for $\alpha \in A(\mathcal{O})$ say, we use the notation $\alpha_{k}=\left.\alpha\right|_{\Omega_{k}}$, and furthermore write without qualification 


$$
\alpha=\left(\alpha_{1}, \ldots, \alpha_{n}\right)
$$

Similarly, we have isomorphisms between $A(\mathcal{O})_{e}$ and $\bigoplus_{k=1}^{n} A\left(\Omega_{k}\right)_{e} ; \mathcal{D}(\mathcal{O})$ and $\bigoplus_{k=1}^{n} \mathcal{D}\left(\Omega_{k}\right)$; and so on.

With this convention in mind, for $\left(\bar{\alpha}_{1}, \ldots, \bar{\alpha}_{n}\right) \in \bigoplus_{k=1}^{n} \overline{A\left(\Omega_{k}\right)}$, observe that $T(\mathcal{O}, \Sigma)$ can be written

$$
\begin{aligned}
{\left[T(\mathcal{O}, \Sigma)\left(\bar{\alpha}_{1}, \ldots, \bar{\alpha}_{n}\right)\right](z) } & :=\frac{1}{\pi i} \sum_{k=1}^{n} \iint_{\Omega_{k}, w} \partial_{z} \partial_{w} \mathscr{G}_{R}(w ; z, q) \wedge \bar{\alpha}_{k}(w) \\
& =\sum_{k=1}^{n}\left[T\left(\Omega_{k}, \Omega_{k}^{*}\right) \bar{\alpha}_{k}\right]_{\Sigma}(z)
\end{aligned}
$$

if $z \in \Sigma$ (recall that $\Omega_{k}^{*}$ is the complement of the closure of $\Omega_{k}$ in $R$ ). Here for a set $A$, by $[\cdot]_{A}$ we mean the restriction to $A$. The above expression shows that $T(\mathcal{O}, \Sigma)$ is bounded as claimed in the introduction. For fixed $j=1, \ldots, n$, we now define

$$
\begin{aligned}
T\left(\mathcal{O}, \Omega_{j}\right): \overline{A(\mathcal{O})} & \rightarrow A\left(\Omega_{j}\right) \\
\left(\bar{\alpha}_{1}, \ldots, \bar{\alpha}_{n}\right) & \mapsto \sum_{\substack{k=1 \\
k \neq j}}^{n}\left[T\left(\Omega_{k}, \Omega_{k}^{*}\right) \bar{\alpha}_{k}\right]_{\Omega_{j}}+T\left(\Omega_{j}, \Omega_{j}\right) \bar{\alpha}_{j} .
\end{aligned}
$$

Again $\left[T\left(\Omega_{k}, \Omega_{k}^{*}\right) \bar{\alpha}_{k}\right]_{\Omega_{j}}$ is the restriction of $T\left(\Omega_{k}, \Omega_{k}^{*}\right) \bar{\alpha}_{k}$ to $\Omega_{j}$. As above, boundedness follows directly from boundedness in the case for one boundary curve. Finally define the bounded operator

$$
\begin{aligned}
S(\mathcal{O}, R): A(\mathcal{O}) & \rightarrow A(R) \\
\alpha & \mapsto \iint_{\mathcal{O}} K_{R}(\cdot, w) \wedge_{w} \alpha(w) .
\end{aligned}
$$

which again can be written as a sum of integrals over $\Omega_{k}$ :

$$
S(\mathcal{O}, R) \alpha=\sum_{k=1}^{n} S\left(\Omega_{k}, R\right) \alpha_{k}
$$

As was observed above, in the case that $R$ is of genus zero, $S(\mathcal{O}, R)=0$.

We also set

$$
\Gamma=\Gamma_{1} \cup \cdots \cup \Gamma_{n}
$$

and define for $\left(h_{1}, \ldots, h_{n}\right) \in \mathcal{D}_{\text {harm }}(\mathcal{O})$ and $z \in R \backslash \Gamma$

$$
\left[J_{q}(\Gamma)\left(h_{1}, \ldots, h_{n}\right)\right](z)=\sum_{k=1}^{n}\left[J_{q}\left(\Gamma_{k}\right) h_{k}\right](z) .
$$


The identities (2.3) can now be generalized as follows.

Theorem 2.2 If $q$ is in $R \backslash \Gamma$ and $\left(h_{1}, \ldots, h_{n}\right) \in \mathcal{D}_{\text {harm }}(\mathcal{O})$ then

$$
\begin{array}{lc}
\partial\left[J_{q}(\Gamma)\left(h_{1}, \ldots, h_{n}\right)\right](z)=-\left[T(\mathcal{O}, \Sigma)\left(\bar{\partial} h_{1}, \ldots, \bar{\partial} h_{n}\right)\right](z), & z \in \Sigma, \\
\partial\left[J_{q}(\Gamma)\left(h_{1}, \ldots, h_{n}\right)\right](z)=-\left[T\left(\mathcal{O}, \Omega_{j}\right)\left(\bar{\partial} h_{1}, \ldots, \bar{\partial} h_{n}\right)\right](z)+\partial h_{j}(z), & z \in \Omega_{j}, \\
\bar{\partial}\left[J_{q}(\Gamma)\left(h_{1}, \ldots, h_{n}\right)\right](z)=\left[\bar{S}(\mathcal{O}, R)\left(\bar{\partial} h_{1}, \ldots, \bar{\partial} h_{n}\right)\right](z) & z \in R \backslash \Gamma .
\end{array}
$$

Proof The first and third identities follow directly from (2.3), (2.5), and (2.7).

Now let $z \in \Omega_{j}$ for fixed $j$; in this case for every $k \neq j, z \in \Omega_{k}^{*}$. Denoting by $J_{q}(\Gamma)_{\Omega_{k}^{*}}$ the operator obtained by restricting the output of $J_{q}(\Gamma)$ to $\Omega_{k}^{*}$, and similarly for $J_{q}(\Gamma)_{\Omega_{j}}$, we have

$$
\begin{aligned}
\partial\left[J_{q}(\Gamma)\left(h_{1}, \ldots, h_{n}\right)\right]_{\Omega_{j}} & =\partial \sum_{k=1}^{n}\left[J_{q}\left(\Gamma_{k}\right) h_{k}\right]_{\Omega_{j}}(z) \\
& =\sum_{k \neq j} \partial\left[J_{q}\left(\Gamma_{k}\right) \Omega_{\Omega_{k}^{*}} h_{k}\right]_{\Omega_{j}}(z)+\partial\left[J_{q}\left(\Gamma_{j}\right)_{\Omega_{j}} h_{j}\right](z) \\
& =\sum_{k \neq j}\left[T\left(\Omega_{k}, \Omega_{k}^{*}\right) \bar{\partial} h_{k}\right]_{\Omega_{j}}(z)+\partial h_{j}(z)+T\left(\Omega_{j}, \Omega_{j}\right) \bar{\partial} h_{j}(z) \\
& =T\left(\mathcal{O}, \Omega_{j}\right)\left(\bar{\partial} h_{1}, \ldots, \bar{\partial} h_{n}\right)(z)+\partial h_{j}(z) .
\end{aligned}
$$

Throughout the paper, we will denote

$$
J_{q}(\Gamma)_{\Sigma} h=\left.J_{q} h\right|_{\Sigma}, \quad J_{q}(\Gamma)_{\mathcal{O}} h=\left.J_{q} h\right|_{\mathcal{O}}, \quad J_{q}(\Gamma)_{\Omega_{k}} h=\left.J_{q} h\right|_{\Omega_{k}}
$$

and so on, as above. Thus, for example, the first two identities of the previous theorem can be expressed by $\partial J_{q}(\Gamma)_{\Sigma}=-T(\mathcal{O}, \Sigma) \bar{\partial}$ and $\partial J_{q}(\Gamma)_{\Omega_{j}}=\partial-T\left(\mathcal{O}, \Omega_{j}\right) \bar{\partial}$.

\subsection{Transmission of harmonic Dirichlet-Bounded Functions}

In this section, we consider certain operators, which take Dirichlet-bounded functions on one region to Dirichlet-bounded functions on another region sharing a portion of the boundary with the first, in such a way that the functions have the same boundary values. These operators were studied in [19]; we briefly recall the necessary results.

We now explain the sense of boundary values, which we call "conformally nontangential boundary values," or CNT boundary values. All claims made here in the description of these boundary values are proven in [19]. For the purposes of this section we can assume that $\mathcal{O}=\Omega$ is a single simply connected domain and $\Omega^{*}$ is the complement of its closure, as above. 
For $p \in \Omega$, let

$$
\Omega_{p, \epsilon}=\left\{w \in \Omega: \mathscr{G}_{\Omega}(w, p)<\epsilon\right\}
$$

Similarly for $p^{\prime} \in \Omega^{*}$, let

$$
\Omega_{p^{\prime}, \epsilon}^{*}=\left\{w \in \Omega^{*}: \mathscr{G}_{\Omega^{*}}\left(w, p^{\prime}\right)<\epsilon\right\} .
$$

For $\epsilon$ sufficiently small, there is a conformal map $f: \mathbb{A} \rightarrow \Omega_{p^{\prime}, \epsilon}^{*}$ where $\mathbb{A}=\{z$ : $\left.e^{-\epsilon}<|z|<1\right\}$, where $f$ extends to a homeomorphism from $\mathbb{S}^{1}$ onto $\Gamma$. We can always assume that $f$ has a conformal extension to a neighborhood of $|z|=e^{-\epsilon}$ so that the image of this curve is analytic. In the case of $\Omega_{p, \epsilon}$, such a conformal map exists for all $\epsilon \in(0,1)$ and is in fact the restriction of the conformal map from $\mathbb{D}$ to $\Omega$ taking 0 to $p$. In particular, $\Omega_{p^{\prime}, \epsilon}^{*}$ and $\Omega_{p, \epsilon}$ are doubly connected domains.

Given a $u \in \mathcal{D}_{\text {harm }}\left(\Omega_{p, \epsilon}\right)$ or $\mathcal{D}_{\text {harm }}\left(\Omega_{p^{\prime}, \epsilon}^{*}\right)$, the non-tangential boundary values of $u \circ f$ exist except perhaps on a Borel set of logarithmic capacity zero. The boundary value of $u$ at a point $\zeta \in \Gamma$ is defined to be the boundary value of $u \circ f$ at $f^{-1}(\zeta)$, where it exists. We call these boundary values the CNT boundary values.

We call the image of a Borel set of logarithmic capacity zero under $f$ a "null set" on $\Gamma$. This definition can be shown to be independent on $p$ or $p^{\prime}$, and the particular choice of map $f$. Similarly, the CNT boundary values are independent of these choices. An important fact is that if $u_{1}, u_{2} \in \mathcal{D}_{\text {harm }}(\Omega)$ have the same CNT boundary values except possibly on a null set, then they are equal. The same holds for $u_{1}, u_{2} \in \mathcal{D}_{\text {harm }}\left(\Omega^{*}\right)$.

It is a much more subtle fact that a set on $\Gamma$ is null with respect to the conformal map onto $\Omega_{p, \epsilon}$ if and only if it is null with respect to the conformal map onto $\Omega_{p^{\prime}, \epsilon}^{*}$, in the case that $\Gamma$ is a quasicircle. More is true: if $u$ is the CNT boundary values of an element of $\mathcal{D}_{\text {harm }}(\Omega)$ except possibly on a null set, then it is the CNT boundary values of a unique element of $\mathcal{D}_{\text {harm }}\left(\Omega^{*}\right)$ except on a null set, and the converse also holds.

This allows us to define an operator that we call the transmission operator. Given $u \in \mathcal{D}_{\text {harm }}(\Omega)$, one may obtain boundary values of $u$ on $\Gamma$ in the CNT sense (see Remark 2.3 below). There exists a unique element of $\mathcal{D}_{\text {harm }}\left(\Omega^{*}\right)$ with the same CNT boundary values, which we denote by $\mathfrak{O}\left(\Omega, \Omega^{*}\right) u$. This defines a map

$$
\mathfrak{O}\left(\Omega, \Omega^{*}\right): \mathcal{D}_{\text {harm }}(\Omega) \rightarrow \mathcal{D}_{\text {harm }}\left(\Omega^{*}\right),
$$

and similarly a map

$$
\mathfrak{O}\left(\Omega^{*}, \Omega\right): \mathcal{D}_{\text {harm }}\left(\Omega^{*}\right) \rightarrow \mathcal{D}_{\text {harm }}(\Omega) .
$$

By definition, these are inverses of each other. It was shown [19] that these are bounded with respect to the Dirichlet seminorm. We call these transmission operators, since in some sense they transmit a harmonic function through the quasicircle via a boundary value problem.

Similarly, the CNT boundary values of any element of $\mathcal{D}_{\text {harm }}\left(\Omega_{p, \epsilon}\right)$ are equal to the CNT boundary values of a unique element of $\mathcal{D}_{\text {harm }}(\Omega)$ up to a null set. The converse 
is obviously true by simply restricting from $\Omega$ to $\Omega_{p, \epsilon}$; however, one does not obtain a unique element of $\mathcal{D}_{\text {harm }}\left(\Omega_{p, \epsilon}\right)$. The same claims are true for $\Omega^{*}$ and $\Omega_{p^{\prime}, \epsilon}^{*}$. This allows us to define the following operator:

$$
\mathfrak{G}\left(\Omega_{p, \epsilon}, \Omega\right): \mathcal{D}_{\text {harm }}\left(\Omega_{p, \epsilon}\right) \rightarrow \mathcal{D}_{\text {harm }}(\Omega)
$$

to take $u$ to the unique element of $\mathcal{D}_{\text {harm }}(\Omega)$ with the same CNT boundary values. Similarly we define

$$
\mathfrak{G}\left(\Omega_{p^{\prime}, \epsilon}^{*}, \Omega^{*}\right): \mathcal{D}_{\text {harm }}\left(\Omega_{p^{\prime}, \epsilon}^{*}\right) \rightarrow \mathcal{D}_{\text {harm }}\left(\Omega^{*}\right)
$$

It was shown in [19] that these are bounded with respect to the Dirichlet seminorm. We call these bounce operators.

The integral (2.1) could equally be defined using level curves in $\Omega^{*}$. That is, let $p^{\prime} \in \Omega^{*}$ and let $\Gamma_{\epsilon}^{p^{\prime}}$ denote the level curves of Green's function, but now give them a negative orientation with respect to $\Omega^{*}$. If we denote by $J_{q}\left(\Gamma, \Omega^{*}\right)$ the new operator defined using negatively oriented level curves in $\Omega^{*}$, and by $J_{q}(\Gamma, \Omega)$ the original operator defined using positively oriented level curves in $\Omega$, then [20, Theorem 4.10]

$$
J_{q}\left(\Gamma, \Omega^{*}\right) \mathfrak{O}\left(\Omega, \Omega^{*}\right)=J_{q}(\Gamma, \Omega) .
$$

The notation $J_{q}(\Gamma)$ will always refer to $J_{q}(\Gamma, \Omega)$. The latter notation is used only when it is necessary to distinguish $J_{q}(\Gamma, \Omega)$ from $J_{q}\left(\Gamma, \Omega^{*}\right)$.

We will also use the following notation for $h \in \mathcal{D}\left(\Omega_{p, \epsilon}\right)$.

$$
J_{q}(\Gamma)^{\prime} h(z)=-\frac{1}{\pi i} \lim _{s \searrow 0} \int_{\Gamma_{s}^{p}} \partial_{w} \mathscr{G}_{R}(w ; z, q) h(w) .
$$

Although the integral is the same, the prime is included to distinguish it from the operator $J_{q}(\Gamma)$, which has a different domain. For any one-form in $A\left(\Omega_{p, \epsilon}\right)$ and $h \in \mathcal{D}_{\text {harm }}\left(\Omega_{p, \epsilon}\right)$, we have [20, Theorem 4.8]

$$
\lim _{s \searrow 0} \int_{\Gamma_{s}^{p}} \alpha h=\lim _{s \searrow 0} \int_{\Gamma_{s}^{p}} \alpha \mathfrak{G}\left(\Omega_{p, \epsilon}, \Omega\right) h .
$$

Similarly [20, Theorem 4.9]

$$
J_{q}(\Gamma)^{\prime} h=J_{q}(\Gamma) \mathfrak{G}\left(\Omega_{p, \epsilon}, \Omega\right) h .
$$

Finally, we define $\mathcal{H}(\Gamma)$ to be the set of complex-valued functions defined on $\Gamma$, which are the CNT boundary values of an element $h \in \mathcal{D}_{\text {harm }}(\Omega)$, modulo the following equivalence relation. We say that $h_{1} \sim h_{2}$ if $h_{1}$ and $h_{2}$ are equal except possibly on a null set. We will continue to treat equivalence classes as functions in the customary way. 
Remark 2.3 Except for Theorem 1.2, the precise meaning of "conformally nontangential" is not directly relevant to the paper; the identities (2.11), (2.13), and (2.14) above are logically sufficient to obtain the results here. Of course, the meaning is helpful for an intuitive understanding of several theorems and proofs.

\subsection{Density Theorems for the Image of $\mathfrak{G}$}

In this section we prove some preliminary density theorems.

Let $R$ be a compact Riemann surface, and $\mathcal{O}, \Omega_{k}$, and $\Gamma_{k}$, for $k=1, \ldots, n$ be as above.

Now recall that

$$
W=\left\{\left(g_{1}, \ldots, g_{n}\right) \in \oplus_{k=1}^{n} \mathcal{D}_{\text {harm }}\left(\Omega_{k}\right): \sum_{k=1}^{n} \iint_{\Omega_{k}} \alpha \wedge \bar{\partial} g_{k}=0 \forall \alpha \in A(R)\right\}
$$

where we have made use of the isomorphism (2.4) to write $W$ in terms of the restrictions $g_{k}$ to $\Omega_{k}$. We also denote

$$
W^{\prime}=\left\{\left(\bar{h}_{1}, \ldots, \bar{h}_{n}\right) \in \oplus_{k=1}^{n} \overline{\mathcal{D}\left(\Omega_{k}\right)}: \sum_{k=1}^{n} \iint_{\Omega_{k}} \alpha \wedge \overline{\partial h}_{k}=0 \forall \alpha \in A(R)\right\} .
$$

Because $\Omega_{k}$ is simply connected, we may decompose any $g$ as $g=e+\bar{h}$ where $e$ has only holomorphic components and $\bar{h}$ has only anti-holomorphic components. Thus one may define $W$ equivalently to be the set of elements $g$ of $\oplus_{k=1}^{n} \mathcal{D}_{\text {harm }}\left(\Omega_{k}\right)$ whose anti-holomorphic component is in $W^{\prime}$.

In the case that $R$ has genus zero, we have that $W=\oplus_{k=1}^{n} \mathcal{D}_{\text {harm }}\left(\Omega_{k}\right)$ and $W^{\prime}=$ $\oplus_{k=1}^{n} \overline{\mathcal{D}\left(\Omega_{k}\right)}$.

Note that

$$
\left(\bar{h}_{1}, \ldots, \bar{h}_{n}\right) \in W^{\prime} \Leftrightarrow \sum_{k=1}^{n} \lim _{s \searrow 0} \int_{\Gamma_{s}^{p_{k}}} \bar{h}_{k} \alpha=0 \quad \forall \alpha \in A(R)
$$

by Stokes' theorem.

Lemma 2.4 If $\left(h_{1}, \ldots, h_{n}\right) \in W$ then $J_{q}(\Gamma)\left(h_{1}, \ldots, h_{n}\right)$ is a holomorphic function on $R \backslash \Gamma$.

Proof We need to show that $\bar{\partial}\left[J_{q}(\Gamma)\left(h_{1}, \ldots, h_{n}\right)\right](z)=0$ for every $z \in R \backslash \Gamma$. We use Theorem 2.2 to derive the first identity below.

$$
\begin{aligned}
\bar{\partial}\left[J_{q}(\Gamma)\left(h_{1}, \ldots, h_{n}\right)\right](z)= & {\left[\bar{S}(\mathcal{O}, R)\left(\bar{\partial} h_{1}, \ldots, \bar{\partial} h_{n}\right)\right](z) } \\
= & \frac{\sum_{k=1}^{n} \iint_{\Omega_{k}, w} K_{R}(z, w) \wedge \partial \bar{h}_{k}(w) .}{}
\end{aligned}
$$


The last integral is zero since $K_{R}(z, \cdot) \in \overline{A(R)}$ for each fixed $z$ and $\left(\bar{\partial} h_{1}, \ldots, \bar{\partial} h_{n}\right) \in$ $V$.

For fixed choice of $p_{k} \in \Omega_{k}, k=1, \ldots, n$, let $\Omega_{k, p_{k}, \epsilon}$ be the domains bounded by level curves of Green's functions of $\Omega_{k}$ as in the previous section. Define now the spaces

$$
X_{\epsilon}=\left\{\left(u_{1}, \ldots, u_{n}\right) \in \oplus_{k} \mathcal{D}\left(\Omega_{k, p_{k}, \epsilon}\right): \sum_{k=1}^{n} \int_{\gamma_{k}} u_{k} \alpha=0 \forall \alpha \in A(R)\right\}
$$

Here, for $k=1, \ldots, n, \gamma_{k}$ is any choice of simple closed analytic curve in $\Omega_{k, \epsilon, p_{k}}$ which is isotopic to $\Gamma_{\epsilon}^{p_{k}}$ within the closure of $\Omega_{k, p_{k}, \epsilon}$. In the case that $R$ has genus zero, $X_{\epsilon}=\oplus_{k} \mathcal{D}\left(\Omega_{k, p_{k}, \epsilon}\right)$.

Since all theorems hold for any choice of $p_{k}$, we will remove the points from the notation for the domains. That is, we will denote $\Omega_{k, p_{k}, \epsilon}$ by $\Omega_{k, \epsilon}$.

Recall the definition (2.10) of the bounce operator. Denote

$$
\oplus_{k} \mathfrak{G}\left(\Omega_{k, \epsilon}, \Omega_{k}\right)\left(u_{1}, \ldots, u_{n}\right)=\left(\mathfrak{G}\left(\Omega_{1, \epsilon}, \Omega_{1}\right) u_{1}, \ldots, \mathfrak{G}\left(\Omega_{n, \epsilon}, \Omega_{n}\right) u_{n}\right)
$$

We then have the following theorem.

Theorem 2.5 Let $\gamma_{k}, k=1, \ldots, n$ be analytic Jordan curves in $\Omega_{k, \epsilon}$, respectively, such that each $\gamma_{k}$ is isotopic to $\Gamma_{\epsilon}^{p_{k}}$ within the closure of $\Omega_{k, \epsilon}$. Given any

$$
\left(u_{1}, \ldots, u_{n}\right) \in \oplus_{k} \mathcal{D}\left(\Omega_{k, \epsilon}\right)
$$

we have that

$$
\oplus_{k} \mathfrak{G}\left(\Omega_{k, \epsilon}, \Omega_{k}\right)\left(u_{1}, \ldots, u_{n}\right) \in W \Leftrightarrow \sum_{k=1}^{n} \int_{\gamma_{k}} u_{k} \alpha=0 \forall \alpha \in A(R)
$$

That is, $\left(u_{1}, \ldots, u_{n}\right) \in X_{\epsilon}$ if and only if $\oplus_{k} \mathfrak{G}\left(\Omega_{k, \epsilon}, \Omega_{k}\right)\left(u_{1}, \ldots, u_{n}\right) \in W$.

Proof This follows directly from (2.13).

Remark 2.6 In the case that the genus of $R$ is zero, Theorem 2.5 is true but vacuous.

We have the following two theorems.

Theorem 2.7 For any $\left(u_{1}, \ldots, u_{n}\right) \in X_{\epsilon}$, the restriction of

$$
\sum_{k=1}^{n} J_{q}\left(\Gamma_{k}\right)^{\prime} u_{k}
$$

to $\Sigma$ extends to a function which is holomorphic on $\mathrm{cl} \Sigma \cup \Omega_{1, \epsilon} \cup \cdots \cup \Omega_{n, \epsilon}$. 
Proof Choose $0<r<\epsilon$. Let $\Gamma_{k, r}$ denote the boundary of $\Omega_{k, r}$. Let $B_{r}$ be the region containing $\Sigma$ and bounded by $\cup_{k=1}^{n} \Gamma_{k, r}$. Applying Royden [14, Proposition 6], together with the explicit integral formula given there, we see that

$$
-\sum_{k=1}^{n} \frac{1}{\pi i} \int_{\Gamma_{k, r}} \partial_{w} \mathscr{G}(w ; z, q) u_{k}(w)
$$

defines a holomorphic function on $B_{r}$. But this integral is independent of $r$ and thus equals the limiting integral (2.12). Since every point in cl $\Sigma \cup \Omega_{1, \epsilon} \cup \cdots \cup \Omega_{n, \epsilon}$ is contained in some $B_{r}$, this proves the theorem.

Corollary 2.8 For any $\left(u_{1}, \ldots, u_{n}\right) \in X_{\epsilon}$, the restriction of

$$
\sum_{k=1}^{n} J_{q}\left(\Gamma_{k}\right) \mathfrak{G}\left(\Omega_{k, \epsilon}, \Omega_{k}\right) u_{k}
$$

to $\Sigma$ has a holomorphic extension to $\mathrm{cl} \Sigma \cup \Omega_{1, \epsilon} \cup \cdots \cup \Omega_{n, \epsilon}$.

Proof This follows immediately from Theorem 2.7 and (2.14).

Remark 2.9 Fix $q \in \Sigma$. Observe that if $\left(g_{1}, \ldots, g_{n}\right)$ and $\left(\hat{g}_{1}, \ldots, \hat{g}_{n}\right)$ are in $W$, and $g_{k}-\hat{g}_{k}$ are holomorphic in $\Omega_{k}$ for all $k$, then

$$
\left.\sum_{k=1}^{n} J_{q}\left(\Gamma_{k}\right) g_{k}\right|_{\Sigma}=\left.\sum_{k=1}^{n} J_{q}\left(\Gamma_{k}\right) \hat{g}_{k}\right|_{\Sigma} .
$$

To see this, by (2.3) we have that

$$
\partial J_{q}\left(\Gamma_{k}\right)_{\Sigma}\left(g_{k}-\hat{g}_{k}\right)=-T\left(\Omega_{k}, \Omega_{k}^{*}\right) \bar{\partial}\left(g_{k}-\hat{g}_{k}\right)=0
$$

on $\Sigma$ for all $k$. Thus $J_{q}\left(\Gamma_{k}\right)\left(g_{k}-\hat{g}_{k}\right)$ is constant on $\Sigma$. Since $J_{q}\left(\Gamma_{k}\right) g_{k}$ and $J_{q}\left(\Gamma_{k}\right) \hat{g}_{k}$ both vanish at $q$, this proves the claim. On the other hand, if $q \notin \Sigma$, then by (2.17) the difference between the left-hand side and right-hand side of (2.16) is constant.

Theorem 2.10 The set $\oplus_{k} \mathfrak{G}\left(\Omega_{k, \epsilon}, \Omega_{k}\right) X_{\epsilon}$ is dense in $W$.

Proof The proof follows the structure of that of Theorem 4.16 in [20], but generalizes it to the case of several boundary curves.

Define $\mathcal{P}: \bigoplus_{k=1}^{n} \mathcal{D}\left(\Omega_{k, \epsilon}\right) \rightarrow X_{\epsilon}$ the orthogonal projection to the subspace $X_{\epsilon}$. Fix a basis $\left\{\alpha_{1}, \ldots, \alpha_{g}\right\}$ for the vector space $A(R)$, where $g$ is the genus of $R$. Define the operator $Q$ by

$$
Q: \bigoplus_{k=1}^{n} \mathcal{D}\left(\Omega_{k, \epsilon}\right) \rightarrow \mathbb{C}^{g}
$$




$$
\left(u_{1}, \ldots, u_{n}\right) \rightarrow\left(\sum_{k=1}^{n} \int_{\Gamma_{k}^{\prime}} u_{k} \alpha_{1}, \ldots, \sum_{k=1}^{n} \int_{\Gamma_{k}^{\prime}} u_{k} \alpha_{g}\right)
$$

For any constants $c_{k} \in \mathbb{C}, k=1, \ldots, n, Q\left(u_{1}+c_{1}, \ldots, u_{n}+c_{n}\right)=Q\left(u_{1}, \ldots, u_{n}\right)$. Furthermore, for any fixed $k$ and fixed $x_{k} \in \Gamma_{k}^{\prime}$ there is a uniform $C$ such that

$$
\sup _{z \in \Gamma_{k}^{\prime}}\left|u_{k}(z)-u_{k}\left(x_{k}\right)\right| \leq C\left\|u_{k}-u_{k}\left(x_{k}\right)\right\|_{\mathcal{D}\left(\Omega_{k, \epsilon}\right)}
$$

These two facts together imply that $Q$ is bounded.

By using the Riesz representation theorem and the Gram-Schmidt process

$\exists C>0$ s.t. $\left\|\mathcal{P}\left(u_{1}, \ldots, u_{n}\right)-\left(u_{1}, \ldots, u_{n}\right)\right\|_{\bigoplus_{k=1}^{n} \mathcal{D}\left(\Omega_{k, \epsilon}\right)} \leq C\left\|Q\left(u_{1}, \ldots, u_{n}\right)\right\|_{\mathbb{C}^{g}}$.

Now define $Q_{1}$ by

$$
\begin{aligned}
Q_{1}: \bigoplus_{k=1}^{n} \mathcal{D}_{\text {harm }}\left(\Omega_{k}\right) & \rightarrow \mathbb{C}^{g} \\
\quad\left(h_{1}, \ldots, h_{n}\right) & \rightarrow\left(\lim _{s \searrow 0^{+}} \sum_{k=1}^{n} \int_{\Gamma_{s}^{p_{k}}} h_{k} \alpha_{1}, \ldots, \lim _{s \searrow 0^{+}} \sum_{k=1}^{n} \int_{\Gamma_{s}^{p_{k}}} h_{k} \alpha_{g}\right) .
\end{aligned}
$$

By the definition of $W, Q_{1}\left(h_{1}, \ldots, h_{n}\right)=0$ if $\left(h_{1}, \ldots, h_{n}\right) \in W$. By Stokes' theorem and continuity of the Dirichlet inner product, there exists $D>0$ such that

$$
\left\|Q_{1}\left(h_{1}, \ldots, h_{n}\right)\right\|_{\mathbb{C}^{g}} \leq D\left\|\left(h_{1}, \ldots, h_{n}\right)\right\|_{\bigoplus_{k=1}^{n}} \mathcal{D}_{\text {harm }}\left(\Omega_{k}\right)
$$

By (2.13)

$$
\begin{aligned}
Q\left(u_{1}, \ldots, u_{n}\right) & =Q_{1}\left(\mathfrak{G}\left(\Omega_{1, \epsilon}, \Omega_{1}\right) u_{1}, \ldots, \mathfrak{G}\left(\Omega_{n, \epsilon}, \Omega_{n}\right) u_{n}\right) \\
& =Q_{1}\left(\oplus_{k} \mathfrak{G}\left(\Omega_{k, \epsilon}, \Omega_{k}\right)\left(u_{1}, \ldots, u_{n}\right)\right)
\end{aligned}
$$

Now let $\left(h_{1}, \ldots, h_{n}\right) \in W$ and $\epsilon>0$, then by density of $\mathfrak{G}\left(\Omega_{k, \epsilon}, \Omega_{k}\right) \mathcal{D}\left(\Omega_{k, \epsilon}\right)$ in $\mathcal{D}_{\text {harm }}\left(\Omega_{k}\right)$ [20, Theorem 4.6], for each $k=1, \ldots, n$ there exists $u_{k} \in \mathcal{D}\left(\Omega_{k, \epsilon}\right)$ such that

$$
\left\|\mathfrak{G}\left(\Omega_{k, \epsilon}, \Omega_{k}\right) u_{k}-h_{k}\right\|_{\mathcal{D}_{\text {harm }}\left(\Omega_{k}\right)} \leq \frac{\epsilon}{\sqrt{n}},
$$

Therefore by the Minkowski inequality we have

$$
\begin{array}{rl}
\| \oplus_{k} & \mathfrak{G}\left(\Omega_{k, \epsilon}, \Omega_{k}\right) \mathcal{P}\left(u_{1}, \ldots, u_{n}\right)-\left(h_{1}, \ldots, h_{n}\right) \| \\
\leq & \left\|\oplus_{k} \mathfrak{G}\left(\Omega_{k, \epsilon}, \Omega_{k}\right) \mathcal{P}\left(u_{1}, \ldots, u_{n}\right)-\oplus_{k} \mathfrak{G}\left(\Omega_{k, \epsilon}, \Omega_{k}\right)\left(u_{1}, \ldots, u_{n}\right)\right\| \\
& +\left\|\oplus_{k} \mathfrak{G}\left(\Omega_{k, \epsilon}, \Omega_{k}\right)\left(u_{1}, \ldots, u_{n}\right)-\left(h_{1}, \ldots, h_{n}\right)\right\| \\
\leq & \left\|\oplus_{k} \mathfrak{G}\left(\Omega_{k, \epsilon}, \Omega_{k}\right)\right\|\left\|\mathcal{P}\left(u_{1}, \ldots, u_{n}\right)-\left(u_{1}, \ldots, u_{n}\right)\right\| \\
& +\left\|\left(\mathfrak{G}\left(\Omega_{1, \epsilon}, \Omega_{1}\right) u_{1}-h_{1}, \ldots, \mathfrak{G}\left(\Omega_{n, \epsilon}, \Omega_{n}\right) u_{n}-h_{n}\right)\right\|
\end{array}
$$


where all the norms are $\|\cdot\|_{\bigoplus_{k=1}^{n}} \mathcal{D}_{\text {harm }}\left(\Omega_{k}\right)$ except the operator norm $\left\|\oplus_{k} \mathfrak{G}\left(\Omega_{k, \epsilon}, \Omega_{k}\right)\right\|$. Since $Q_{1}\left(h_{1}, \ldots, h_{n}\right)=0$ one has

$$
\begin{aligned}
\left\|\mathcal{P}\left(u_{1}, \ldots, u_{n}\right)-\left(u_{1}, \ldots, u_{n}\right)\right\| & \leq C\left\|Q\left(u_{1}, \ldots, u_{n}\right)\right\|_{\mathbb{C}^{g}} \\
& =C\left\|Q_{1} \oplus_{k} \mathfrak{G}\left(\Omega_{k, \epsilon}, \Omega_{k}\right)\left(u_{1}, \ldots, u_{n}\right)\right\|_{\mathbb{C}^{g}} \\
& =C \| Q_{1}\left(\oplus_{k} \mathfrak{G}\left(\Omega_{k, \epsilon}, \Omega_{k}\right)\left(u_{1}, \ldots, u_{n}\right)\right. \\
& \left.-\left(h_{1}, \ldots, h_{n}\right)\right) \|_{\mathbb{C}^{g}} \\
& \leq C D\left\|\oplus_{k} \mathfrak{G}\left(\Omega_{k, \epsilon}, \Omega_{k}\right)\left(u_{1}, \ldots, u_{n}\right)-\left(h_{1}, \ldots, h_{n}\right)\right\| .
\end{aligned}
$$

By our choice of $u_{k}$ 's for the second term we have

$$
\begin{aligned}
\| & \left(\mathfrak{G}\left(\Omega_{1, \epsilon}, \Omega_{1}\right) u_{1}-h_{1}, \ldots, \mathfrak{G}\left(\Omega_{n, \epsilon}, \Omega_{n}\right) u_{n}-h_{n}\right) \| \\
= & \left(\sum_{k=1}^{n}\left\|\mathfrak{G}\left(\Omega_{k, \epsilon}, \Omega_{k}\right) u_{k}-h_{k}\right\|_{\mathcal{D}_{\text {harm }}\left(\Omega_{k}\right)}^{2}\right)^{\frac{1}{2}} \\
& \leq\left(\sum_{k=1}^{n} \frac{\epsilon^{2}}{n}\right)^{\frac{1}{2}}=\epsilon .
\end{aligned}
$$

Combining the above two inequalities with (2.18) we obtain

$$
\begin{aligned}
\| & \oplus_{k} \mathfrak{G}\left(\Omega_{k, \epsilon}, \Omega_{k}\right) \mathcal{P}\left(u_{1}, \ldots, u_{n}\right)-\left(h_{1}, \ldots, h_{n}\right) \| \\
& \leq C D\left\|\oplus_{k} \mathfrak{G}\left(\Omega_{k, \epsilon}, \Omega_{k}\right)\right\|\left\|\oplus_{k} \mathfrak{G}\left(\Omega_{k, \epsilon}, \Omega_{k}\right)\left(u_{1}, \ldots, u_{n}\right)-\left(h_{1}, \ldots, h_{n}\right)\right\|+\epsilon \\
& \leq C D\left\|\oplus_{k} \mathfrak{G}\left(\Omega_{k, \epsilon}, \Omega_{k}\right)\right\| \epsilon+\epsilon=\left(C D\left\|\oplus_{k} \mathfrak{G}\left(\Omega_{k, \epsilon}, \Omega_{k}\right)\right\|+1\right) \epsilon .
\end{aligned}
$$

Therefore $\oplus_{k} \mathfrak{G}\left(\Omega_{k, \epsilon}, \Omega_{k}\right) X_{\epsilon}$ is dense in $W$.

Remark 2.11 In the case that the genus is zero, some of the steps in the proof of Theorem 2.10 are automatic.

\subsection{Proof of Theorem 1.1}

The proof proceeds in several steps.

We will show that the Schiffer operator is a bounded isomorphism on the subspace $V$ of $\bigoplus_{k=1}^{n} \overline{A\left(\Omega_{k}\right)}$. Recall that

$$
V=\left\{\left(\bar{\alpha}_{1}, \ldots, \bar{\alpha}_{n}\right) \in \bigoplus_{k=1}^{n} \overline{A\left(\Omega_{k}\right)}: \sum_{k=1}^{n} \iint_{\Omega_{k}} \beta \wedge \bar{\alpha}_{k}=0 ; \forall \beta \in A(R)\right\}
$$

where we have made use of the isomorphism (2.4) to rewrite $V$ in terms of the restrictions to $\Omega_{k}$. We record the following obvious fact. 
Lemma 2.12 The operator

$$
\begin{aligned}
\bar{\partial}: W^{\prime} & \rightarrow V \\
\left(\bar{h}_{1}, \ldots, \bar{h}_{n}\right) & \rightarrow\left(\overline{\partial h}_{1}, \ldots, \overline{\partial h}_{n}\right),
\end{aligned}
$$

is a surjective operator which preserves the norm.

For fixed $j=1, \ldots, n$ we will define the transmission operator $\mathfrak{O}\left(\Sigma, \Omega_{j}\right)$ from $\Sigma$ to $\Omega_{j}$. Recall that $\Omega_{j}^{*}$ is the complement of the closure of $\Omega_{j}$ in $R$, which contains $\Sigma$. For fixed $p_{j}^{\prime} \in \Omega_{j}^{*}$, let $\Omega_{j, p_{j}^{\prime}, \epsilon}^{*}$ be a doubly connected domain bounded by $\Gamma_{j}$ in $\Sigma$ as in (2.9). Let $\operatorname{Res}\left(\Sigma, \Omega_{j, p_{j}^{\prime}, \epsilon}^{*}\right)$ be the restriction operator from $\mathcal{D}(\Sigma)$ to $\mathcal{D}\left(\Omega_{j, p_{j}^{\prime}, \epsilon}^{*}\right)$. We then define

Definition $2.13 \mathfrak{O}\left(\Sigma, \Omega_{j}\right):=\mathfrak{O}\left(\Omega_{j}^{*}, \Omega_{j}\right) \mathfrak{G}\left(\Omega_{j, p_{j}^{\prime}, \epsilon}^{*}, \Omega_{j}^{*}\right) \operatorname{Res}\left(\Sigma, \Omega_{j, p_{j}^{\prime}, \epsilon}^{*}\right)$.

The interpretation of $\mathfrak{O}\left(\Sigma, \Omega_{j}\right)$ is that it takes elements of $\mathcal{D}_{\text {harm }}(\Sigma)$ to elements of $\mathcal{D}_{\text {harm }}\left(\Omega_{j}\right)$ with the same CNT boundary values on $\Gamma_{j}$; in other words, it is a transmission operator from $\Sigma$ to $\Omega_{j}$. As above, this is independent of the choice of $p_{j}^{\prime}$ [19]. The above expression establishes that the operator is bounded, since each operator on the right-hand side is bounded. Finally, let

$$
\begin{aligned}
\mathfrak{O}(\Sigma, \mathcal{O}): \mathcal{D}_{\text {harm }}(\Sigma) & \rightarrow \bigoplus_{k=1}^{n} \mathcal{D}_{\text {harm }}\left(\Omega_{k}\right) \\
h & \mapsto\left(\mathfrak{O}\left(\Sigma, \Omega_{1}\right) h, \ldots, \mathfrak{O}\left(\Sigma, \Omega_{n}\right) h\right) .
\end{aligned}
$$

Theorem 2.14 For all $\bar{h}=\left(\bar{h}_{1}, \ldots, \bar{h}_{n}\right) \in W^{\prime}$, we have

$$
-\mathfrak{O}\left(\Sigma, \Omega_{j}\right)\left[J_{q}(\Gamma) \bar{h}\right]_{\Sigma}=\bar{h}_{j}-\left[J_{q}(\Gamma) \bar{h}\right]_{\Omega_{j}}
$$

for $j=1, \ldots, n$. That is

$$
-\mathfrak{O}(\Sigma, \mathcal{O})\left[J_{q}(\Gamma) \bar{h}\right]_{\Sigma}=\bar{h}-\left[J_{q}(\Gamma) \bar{h}\right]_{\mathcal{O}}
$$

Proof Since every operator in the identity above is bounded, it suffices to prove this for $\oplus_{k} \mathfrak{G}\left(\Omega_{k, \epsilon}, \Omega_{k}\right) X_{\epsilon}$, because this set is dense in W by Theorem 2.10.

For each $k=1,2, \ldots, n$, let $\Omega_{k, \epsilon}$ be as above in $\Omega_{k}$, and let $h_{k} \in \mathcal{D}\left(\Omega_{k, \epsilon}\right)$. We will apply [14, Theorem 4] with $\mathcal{E}=\cup_{k=1}^{n}\left(\Omega_{k} \backslash \Omega_{k, \epsilon}\right)$ and $\mathcal{O}=\cup_{k=1}^{n} \Omega_{k}$ which are a closed subset and an open subset of $R$, respectively. We also have $\mathcal{O} \backslash \mathcal{E}=\cup_{k=1}^{n} \Omega_{k, \epsilon}$.

Let $h=\left(h_{1}, \ldots, h_{n}\right) \in \bigoplus_{k=1}^{n} \mathcal{D}\left(\Omega_{k, \epsilon}\right)$ satisfying the integral condition

$$
\sum_{k=1}^{n} \int_{\Gamma_{k}^{\prime}} h_{k} \alpha=0 ; \forall \alpha \in A(R),
$$

where for each $k, \Gamma_{k}^{\prime}$ is an analytic curve in $\Omega_{k, \epsilon}$ isotopic to $\Gamma_{k}$ in the closure of $\Omega_{k, \epsilon}$. 
By [14, Theorem 4], there exists $H_{1} \in \mathcal{D}(\mathcal{O})\left(F-f\right.$ in [14]) and $H_{2} \in \mathcal{D}(R \backslash \mathcal{E})=$ $\mathcal{D}\left(\operatorname{cl}(\Sigma) \cup\left(\cup_{k=1}^{n} \Omega_{k, \epsilon}\right)\right)(-f$ in [14]) which satisfy

$$
h(z)=H_{1}(z)-H_{2}(z) ; \forall z \in \mathcal{O} \backslash \mathcal{E}=\cup_{k=1}^{n} \Omega_{k, \epsilon} .
$$

These are explicitly given by the following formulas. Setting $\Gamma^{\prime}=\cup_{k=1}^{n} \Gamma_{k}^{\prime}$ for $z \in$ $\Omega_{j}(\subset \mathcal{O})$, we have

$$
\begin{aligned}
& \left.H_{1}\right|_{\Omega_{j}}(z)=\left[J_{q}\left(\Gamma^{\prime}\right)^{\prime} h\right]_{\mathcal{O}}(z)=-\frac{1}{\pi i} \sum_{k=1}^{n} \int_{\Gamma_{k}^{\prime}} \partial_{w} \mathscr{G}(w ; z, q) h_{k}(w) \\
& =\sum_{k \neq j}\left[J_{q}\left(\Gamma_{k}^{\prime}\right)^{\prime} h_{k}\right]_{\Omega_{j}}(z)+\left[J_{q}\left(\Gamma_{j}^{\prime}\right)^{\prime} h_{j}\right]_{\Omega_{j}}(z)
\end{aligned}
$$

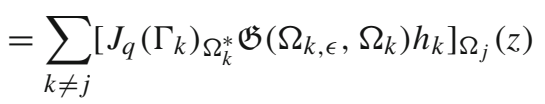

$$
\begin{aligned}
& +\left[J_{q}\left(\Gamma_{j}\right) \mathfrak{G}\left(\Omega_{j, \epsilon}, \Omega_{j}\right) h_{j}\right]_{\Omega_{j}}(z)
\end{aligned}
$$

where the last equality stems from [20, Theorem 4.10]. Applying [20, Theorem 4.10] again, we have for $z \in \Sigma$

$$
\begin{aligned}
\left.H_{2}\right|_{\Sigma}(z) & =-\frac{1}{\pi i} \sum_{k=1}^{n} \int_{\Gamma_{k}^{\prime}} \partial_{w} \mathscr{G}(w ; z, q) h_{k}(w) \\
& =\sum_{k=1}^{n}\left[J_{q}\left(\Gamma_{k}^{\prime}\right)^{\prime} h_{k}\right]_{\Sigma}(z) \\
& =\sum_{k=1}^{n}\left[J_{q}\left(\Gamma_{k}\right)_{\Omega_{k}^{*}} \mathfrak{G}\left(\Omega_{k, \epsilon}, \Omega_{k}\right) h_{k}\right]_{\Sigma}(z)
\end{aligned}
$$

Restricting (2.19) to $\Omega_{j, \epsilon}$ yields

$$
h_{j}(z)=\left.h\right|_{\Omega_{j, \epsilon}}(z)=\left.H_{1}\right|_{\Omega_{j, \epsilon}}(z)-\left.H_{2}\right|_{\Omega_{j, \epsilon}}(z) ; \forall z \in \Omega_{j} .
$$

Since we have

$$
\begin{aligned}
\mathfrak{G}\left(\Omega_{j, \epsilon}, \Omega_{j}\right)\left(\left.H_{1}\right|_{\Omega_{j, \epsilon}}\right) & =H_{1}, \\
\mathfrak{G}\left(\Omega_{j, \epsilon}, \Omega_{j}\right)\left(\left.H_{2}\right|_{\Omega_{j, \epsilon}}\right) & =\mathfrak{O}\left(\Sigma, \Omega_{j}\right)\left(\left.H_{2}\right|_{\Sigma}\right),
\end{aligned}
$$

applying $\mathfrak{G}\left(\Omega_{j, \epsilon}, \Omega_{j}\right)$ to each term of (2.20) we obtain

$$
\mathfrak{G}\left(\Omega_{j, \epsilon}, \Omega_{j}\right) h_{j}(z)=\left.H_{1}\right|_{\Omega_{j}}(z)-\mathfrak{O}\left(\Sigma, \Omega_{j}\right)\left(\left.H_{2}\right|_{\Sigma}\right)(z) ; \forall z \in \Omega_{j}
$$


Finally, inserting the formulas for $H_{1}$ and $H_{2}$ yields that

$$
\begin{aligned}
& \mathfrak{G}\left(\Omega_{j, \epsilon}, \Omega_{j}\right) h_{j}(z)=\sum_{k \neq j}\left[J_{q}\left(\Gamma_{k}\right)_{\Omega_{k}^{*}} \mathfrak{G}\left(\Omega_{k, \epsilon}, \Omega_{k}\right) h_{k}\right]_{\Omega_{j}}(z) \\
& +\left[J_{q}\left(\Gamma_{j}\right) \mathfrak{G}\left(\Omega_{j, \epsilon}, \Omega_{j}\right) h_{j}\right]_{\Omega_{j}}(z) \\
& -\mathfrak{O}\left(\Sigma, \Omega_{j}\right) \sum_{k=1}^{n}\left[J_{q}\left(\Gamma_{k}\right)_{\Omega_{k}^{*}} \mathfrak{G}\left(\Omega_{k, \epsilon}, \Omega_{k}\right) h_{k}\right]_{\Sigma}(z) \quad ; \quad \forall z \in \Omega_{j}
\end{aligned}
$$

which completes the proof.

Remark 2.15 It is easily seen that this holds trivially for all holomorphic $h \in W$, since the left-hand side vanishes by Remark 2.9. Thus the theorem holds for all $h \in W$.

Lemma 2.16 If $h \in \mathcal{D}(\Sigma)_{q}$ then

$$
-h=J_{q}(\Gamma)_{\Sigma} \mathfrak{O}(\Sigma, \mathcal{O}) h
$$

and

$$
0=J_{q}(\Gamma) \mathcal{O} \mathfrak{O}(\Sigma, \mathcal{O}) h
$$

Proof We will distinguish $J_{q}$ defined by limiting integrals from within $\Omega_{k}$ and from within $\Omega_{k}^{*}$ in this proof. Recall that

$$
J_{q}(\Gamma)_{\Sigma} \mathfrak{O}(\Sigma, \mathcal{O}) h=\sum_{k=1}^{n}\left[J_{q}\left(\Gamma_{k}\right) \mathfrak{O}\left(\Sigma, \Omega_{k}\right) h\right]_{\Sigma}
$$

Since the function $h$ is holomorphic on $\Sigma$ it is equal to the sum of the limiting integrals from within $\Omega_{k}^{*}$ for the boundary curves $\Gamma_{k}$. Explicitly, for $z \in \Sigma$, using the residue theorem and the fact that $\partial_{w} \mathscr{G}_{R}$ is holomorphic in $w$ except for simple poles at $z$ and $q$ with residues -1 and 1 , respectively,

$$
-h(z)=-\lim _{s \searrow 0} \frac{1}{\pi i} \sum_{k=1}^{n} \int_{\Gamma_{s}^{k}} \partial_{w} \mathscr{G}_{R}(w ; z, q) h(w) d w .
$$

Note that the curves $\Gamma_{s}^{k}$ are negatively oriented with respect to $\Sigma$. Similarly, for $z \in \mathcal{O}$,

$$
0=-\lim _{s \searrow 0} \frac{1}{\pi i} \sum_{k=1}^{n} \int_{\Gamma_{s}^{k}} \partial_{w} \mathscr{G}_{R}(w ; z, q) h(w) d w .
$$

Let $\Omega_{k, \epsilon}^{*}$ be the doubly connected domain bounded by $\Gamma_{k}$ in $\Sigma$. One can replace each integral in the sum by an integral over a fixed analytic curve $\Gamma_{k}^{\prime}$ in $\Omega_{k, \epsilon}^{*}$. That 
defines an operator $J_{q}\left(\Gamma_{k}, \Omega_{k, \epsilon}^{*}\right)^{\prime}$. For every $k=1, \ldots, n$, [20, Theorem 4.9] yields that

$$
J_{q}\left(\Gamma_{k}, \Omega_{k, \epsilon}^{*}\right)_{\Sigma}^{\prime} h=J_{q}\left(\Gamma_{k}, \Omega_{k}^{*}\right)_{\Sigma}\left[\mathfrak{G}\left(\Omega_{k, \epsilon}^{*}, \Omega_{k}^{*}\right)\left(\left.h\right|_{\Omega_{k, \epsilon}^{*}}\right)\right]
$$

Now apply [20, Theorem 4.10] for each fixed curve $\Gamma_{k}$ and function $\mathfrak{G}\left(\Omega_{k, \epsilon}^{*}, \Omega_{k}^{*}\right)$ $\left(\left.h^{*}\right|_{\Omega_{k, \epsilon}}\right)$ to obtain that

$J_{q}\left(\Gamma_{k}, \Omega_{k}^{*}\right)_{\Sigma}\left[\mathfrak{G}\left(\Omega_{k, \epsilon}^{*}, \Omega_{k}^{*}\right)\left(\left.h\right|_{\Omega_{k, \epsilon}^{*}}\right)\right]=J_{q}\left(\Gamma_{k}, \Omega_{k}\right)_{\Sigma} \mathfrak{O}\left(\Omega_{k}^{*}, \Omega_{k}\right)\left[\mathfrak{G}\left(\Omega_{k, \epsilon}^{*}, \Omega_{k}^{*}\right)\left(\left.h\right|_{\Omega_{k, \epsilon}^{*}}\right)\right]$.

Finally, taking a sum over all terms and using definition 2.13 yield that

$$
\begin{aligned}
-h & =\sum_{k=1}^{n}\left[J_{q}\left(\Gamma_{k}, \Omega_{k, \epsilon}^{*}\right)_{\Sigma}^{\prime}\left(\left.h\right|_{\Omega_{k, \epsilon}^{*}}\right)\right] \\
& =\sum_{k=1}^{n} J_{q}\left(\Gamma_{k}, \Omega_{k}\right)_{\Sigma} \mathfrak{O}\left(\Omega_{k}^{*}, \Omega_{k}\right)\left[\mathfrak{G}\left(\Omega_{k, \epsilon}^{*}, \Omega_{k}^{*}\right)\left(\left.h\right|_{\Omega_{k, \epsilon}^{*}}\right)\right] \\
& =\sum_{k=1}^{n} J_{q}\left(\Gamma_{k}, \Omega_{k}\right)_{\Sigma}\left[\mathfrak{O}\left(\Sigma, \Omega_{k}\right) h\right]
\end{aligned}
$$

which proves the first claim. The second claim is proven by replacing the subscript $\Sigma$ by $\mathcal{O}$ on all $J_{q}$ and $J_{q}^{\prime}$ operators and using (2.22) in place of (2.21).

Theorem 2.17 $T(\mathcal{O}, \Sigma)$ is a surjective operator from $V$ onto $A(\Sigma)_{e}$.

Proof First we show that $T(\mathcal{O}, \Sigma)(V) \subset A(\Sigma)_{e}$.

Let $\bar{\alpha}=\left(\bar{\alpha}_{1}, \ldots, \bar{\alpha}_{n}\right) \in V$. By Lemma 2.12 there is an $\bar{H}=\left(\bar{H}_{1}, \ldots, \bar{H}_{n}\right) \in$ $W^{\prime}$ such that $\bar{\alpha}=\overline{\partial H}$. Moreover, Lemma 2.4 and Remark 2.9 yield that $J_{q}(\Gamma)\left(\bar{H}_{1}, \ldots, \bar{H}_{n}\right)$ is holomorphic. Therefore for $z \in \Sigma$, Theorem 2.2 yields

$$
\begin{aligned}
T(\mathcal{O}, \Sigma)\left(\bar{\alpha}_{1}, \ldots, \bar{\alpha}_{n}\right) & =\partial J_{q}(\Gamma)_{\Sigma}\left(\bar{H}_{1}, \ldots, \bar{H}_{n}\right) \\
& =d J_{q}(\Gamma)_{\Sigma}\left(\bar{H}_{1}, \ldots, \bar{H}_{n}\right) \in A(\Sigma)_{e}
\end{aligned}
$$

Next we show that every element in $A(\Sigma)_{e}$ is in the image of $T(\mathcal{O}, \Sigma)$. Given $\beta \in A(\Sigma)_{e}$, then there exists an $h_{\Sigma} \in \mathcal{D}(\Sigma)_{q}$ such that $\partial_{z} h_{\Sigma}=\beta$. Let $h_{k} \in \mathcal{D}_{\text {harm }}\left(\Omega_{k}\right)$ be such that $\mathfrak{O}\left(\Sigma, \Omega_{k}\right) h_{\Sigma}=h_{k}$, i.e., $h_{\Sigma}$ and $h_{k}$ have the same CNT boundary values on $\Gamma_{k}$. Lemma 2.16 and Theorem 2.2 now imply that

$$
\begin{aligned}
\beta & =\partial h_{\Sigma}=-\partial \sum_{k=1}^{n} J_{q}\left(\Gamma_{k}, \Omega_{k}\right)_{\Sigma}\left[\mathfrak{O}\left(\Sigma, \Omega_{k}\right) h_{\Sigma}\right] \\
& =-\partial J_{q}(\Gamma)_{\Sigma}\left(h_{1}, \ldots, h_{n}\right) \\
& =T(\mathcal{O}, \Sigma)\left(\bar{\partial} h_{1}, \ldots, \bar{\partial} h_{n}\right) .
\end{aligned}
$$


So we need only show that $\left(\bar{\partial} h_{1}, \ldots, \bar{\partial} h_{n}\right)$ is in $V$; that is, for all $\bar{\alpha} \in \overline{A(R)}$,

$$
\sum_{k=1}^{n} \iint_{\Omega_{k}, w} \alpha \wedge \bar{\partial} h_{k}=0
$$

To see this we have

$$
\begin{aligned}
& \sum_{k=1}^{n} \iint_{\Omega_{k}, w} \alpha \wedge \bar{\partial} h_{k}=\sum_{k=1}^{n} \iint_{\Omega_{k}, w} \alpha(w) \wedge \bar{\partial} h_{k}(w) \\
& =\sum_{k=1}^{n} \iint_{\Omega_{k}, w}\left(\iint_{R, z} K_{R}(w, z) \wedge_{z} \alpha(z)\right) \wedge_{w} \bar{\partial} h_{k}(w) \\
& =\sum_{k=1}^{n} \iint_{R, z} \alpha(z) \wedge_{z}\left(\iint_{\Omega_{k}, w} \overline{K_{R}(z, w)} \wedge_{w} \bar{\partial} h_{k}(w)\right) \\
& =\sum_{k=1}^{n} \iint_{R, z} \alpha(z) \wedge_{z}\left(\iint_{\Omega_{k}, w} \bar{\partial}_{z} \partial_{w} g(w ; z, q) \wedge_{w} \bar{\partial} h_{k}(w)\right) \\
& =\iint_{R, z} \alpha(z) \wedge_{z}\left(\sum_{k=1}^{n} \iint_{\Omega_{k}, w} \bar{\partial}_{z} \partial_{w} g(w ; z, q) \wedge_{w} \bar{\partial} h_{k}(w)\right) .
\end{aligned}
$$

Using Lemma 2.16 once again, we have

$$
h_{\Sigma}=-\frac{1}{\pi i} \sum_{k=1}^{n} \iint_{\Omega_{k}, w} \partial_{w} \mathscr{G}(w ; z, q) \wedge_{w} \bar{\partial} h_{k}(w) .
$$

On the other hand, $h_{\Sigma}$ is holomorphic so $\bar{\partial}_{z} h_{\Sigma}=0$. Therefore

$$
\sum_{k=1}^{n} \iint_{\Omega_{k}, w} \bar{\partial}_{z} \partial_{w} \mathscr{G}(w ; z, q) \wedge_{w} \bar{\partial} h_{k}(w)=0
$$

which inserted in (2.23) completes the proof.

We note that the transmission operator $\mathfrak{O}(\Sigma, \Omega)$ induces a transmission on the set of exact forms by conjugating by differentiation. Namely, for fixed $k$ set

$$
\begin{aligned}
\mathfrak{O}_{e}\left(\Sigma, \Omega_{k}\right) & =d \mathfrak{O}\left(\Sigma, \Omega_{k}\right) d^{-1}: A(\Sigma)_{e} \rightarrow A\left(\Omega_{k}\right)_{e} \\
\mathfrak{O}_{e}(\Sigma, \mathcal{O}) & =d \mathfrak{O}(\Sigma, \mathcal{O}) d^{-1}: A(\Sigma)_{e} \rightarrow A(\mathcal{O})_{e}
\end{aligned}
$$

Although $d^{-1}$ is not well defined because of the arbitrary choice of constant, $\mathfrak{O}_{e}\left(\Sigma, \Omega_{k}\right)$ is well defined because $\mathfrak{O}\left(\Sigma, \Omega_{k}\right)$ takes constants to constants. 
Defining

$$
\begin{aligned}
T(\mathcal{O}, \mathcal{O}): \overline{A(\mathcal{O})} & \rightarrow A(\mathcal{O}) \\
\alpha & \mapsto \frac{1}{\pi i} \iint_{\mathcal{O}} \partial_{z} \partial_{w} \mathscr{G}_{R}(w ; z, q) \wedge_{w} \overline{\alpha(w)} \quad z \in \mathcal{O}
\end{aligned}
$$

we then have the following version of Theorem 2.14 for one-forms.

Theorem 2.18 For all $\bar{\alpha}=\left(\bar{\alpha}_{1}, \ldots, \bar{\alpha}_{n}\right) \in V$,

$$
\mathfrak{O}_{e}\left(\Sigma, \Omega_{j}\right) T(\mathcal{O}, \Sigma) \bar{\alpha}=\bar{\alpha}_{j}+T\left(\mathcal{O}, \Omega_{j}\right) \bar{\alpha}
$$

for $j=1, \ldots, n$. That is

$$
\mathfrak{O}_{e}(\Sigma, \mathcal{O}) T(\mathcal{O}, \Sigma) \bar{\alpha}=\bar{\alpha}+T(\mathcal{O}, \mathcal{O}) \bar{\alpha}
$$

Proof This follows from Theorems 2.2 and 2.14.

Now for any open set $D$ of $R$ let $P_{A}(D): A_{\text {harm }}(D) \rightarrow A(D)$ and $\bar{P}_{A}(D)$ : $A_{\text {harm }}(D) \rightarrow \overline{A(D)}$ denote the orthogonal projections.

Corollary $2.19 T(\mathcal{O}, \Sigma)$ is injective on $V$, with left inverse $\bar{P}_{A}(\mathcal{O}) \mathfrak{O}_{e}(\Sigma, \mathcal{O})$.

Proof Apply $\bar{P}_{A}(\mathcal{O})$ to both sides of the second equation of Theorem 2.18.

Now observe that Theorem 1.1 follows directly from Theorem 2.17 and Corollary 2.19 .

\section{Applications of the Isomorphism Theorem}

\subsection{Plemelj-Sokhtoski Jump Problem for Finitely Many Quasicircles}

In this section we establish a jump formula for $n$ quasicircles. Setting aside analytic issues momentarily, the problem is as follows. Given a function $u$ on $\Gamma=\Gamma_{1} \cup \cdots \cup \Gamma_{n}$, find holomorphic functions $u_{k}$ on $\Omega_{k}$ and $u_{\Sigma}$ on $\Sigma$ such that on each curve $\Gamma_{k}$ their boundary values $\tilde{u}_{k}$ and $\tilde{u}_{\Sigma}$ satisfy

$$
\tilde{u}_{k}-\tilde{u}_{\Sigma}=u \text {. }
$$

The solution to this problem is well known for more regular curves, say for $\Gamma$ and $u$ smooth. Here, $\Gamma_{k}$ are of course quasicircles. We consider the class of functions $\mathcal{H}\left(\Gamma_{k}\right)$; recall that these functions are CNT boundary values of elements of $\mathcal{D}_{\text {harm }}\left(\Omega_{k}\right)$.

It is classically known [6], [15] that the topological condition for existence of a solution to the jump problem for functions $u$ on $\Gamma$ is that

$$
\sum_{k=1}^{n} \int_{\Gamma} \alpha u=0
$$


for all one-forms $\alpha \in A(R)$. On quasicircles, this integral condition would not make sense, because quasicircles need not be rectifiable. Thus, we replace this by the condition that $u$ is the boundary values of an element of $W$, motivated by (2.15).

Our first theorem in some sense is the derivative of the jump isomorphism. Let

$$
V^{\prime}=\left\{\bar{\alpha}+\beta \in A_{\text {harm }}(\mathcal{O}): \bar{\alpha} \in V \text { and } \beta \in A(\mathcal{O})\right\}
$$

If $R$ has genus zero, then $V^{\prime}=A_{\text {harm }}(\mathcal{O})$. In reading the theorems below, recall that in this case we also have $V=\overline{A(\mathcal{O})}, W=\mathcal{D}_{\text {harm }}(\mathcal{O})$, and $W^{\prime}=\overline{\mathcal{D}(\mathcal{O})}$.

Theorem 3.1 Let $R$ be a compact Riemann surface, and $\Omega_{1}, \ldots, \Omega_{n}$ be simply connected regions in $R$, bounded by quasicircles $\Gamma_{1}, \ldots, \Gamma_{n}$. Assume that the closures of $\Omega_{1}, \ldots, \Omega_{n}$ are pairwise disjoint. Then

$$
\begin{aligned}
\hat{\mathfrak{H}}: V^{\prime} & \rightarrow A(\mathcal{O}) \oplus A(\Sigma)_{e} \\
\bar{\alpha}+\beta & \mapsto(\beta-T(\mathcal{O}, \mathcal{O}) \bar{\alpha},-T(\mathcal{O}, \Sigma) \bar{\alpha})
\end{aligned}
$$

is a bounded isomorphism.

Proof First we show that $\hat{\mathfrak{H}}$ is injective. Assume that $\hat{\mathfrak{H}}(\bar{\alpha}+\beta)=0$, then $\bar{\alpha}=0$ by Theorem 1.1. But since $0=\beta-T(\mathcal{O}, \mathcal{O}) \bar{\alpha}=\beta$, we also have that $\beta=0$.

Now we show that $\hat{\mathfrak{H}}$ is surjective. Let $\left(\beta_{\mathcal{O}}, \beta_{\Sigma}\right) \in A(\mathcal{O}) \oplus A(\Sigma)_{e}$. By Theorem 1.1 there is an $\bar{\alpha} \in V$ such that $-T(\mathcal{O}, \Sigma) \bar{\alpha}=\beta_{\Sigma}$. Setting $\beta=\beta_{\mathcal{O}}+T(\mathcal{O}, \mathcal{O}) \bar{\alpha}$ yields that $\hat{\mathfrak{H}}(\beta+\bar{\alpha})=\left(\beta_{\mathcal{O}}, \beta_{\Sigma}\right)$.

Theorem 3.2 Let $R, \mathcal{O}$, and $\Sigma$ be as in Theorem 3.1. Then

$$
\begin{aligned}
\mathfrak{H}: \mathcal{D}_{\text {harm }}(\mathcal{O}) & \rightarrow \mathcal{D}(\mathcal{O}) \oplus \mathcal{D}(\Sigma)_{q} \\
h & \mapsto\left(\left.J_{q}(\Gamma) h\right|_{\mathcal{O}},\left.J_{q}(\Gamma) h\right|_{\Sigma}\right)
\end{aligned}
$$

is a bounded isomorphism from $W$ to $\mathcal{D}(\mathcal{O}) \oplus \mathcal{D}(\Sigma)_{q}$.

Proof By Lemma 2.4 the image of $\mathfrak{H}$ is in $\mathcal{D}(\mathcal{O}) \oplus \mathcal{D}(\Sigma)$. By (2.1), the output vanishes at $q$. Thus the image of $\mathfrak{H}$ is in $\mathcal{D}(\mathcal{O}) \oplus \mathcal{D}(\Sigma)_{q}$.

By Theorem 2.2, $\partial \mathfrak{H}=\hat{\mathfrak{H} d}$ when restricted to $W$. Assume that $h \in W$ satisfies $\mathfrak{H} h=0$. Then $\hat{\mathfrak{H}} d h=0$, so $d h=0$, so $h$ is constant on $\mathcal{O}$. But if $h$ is a constant $c$ then $\left.J_{q}(\Gamma) c\right|_{\Sigma}=c$. Since $c \in \mathcal{D}(\Sigma)_{q}$ it vanishes at $q$, so $c=0$. So $\mathfrak{H}$ is injective.

We also have that $\mathfrak{H}(h+c)=\mathfrak{H} h+(c, 0)$ for any constant $c$. This together with the fact that $\hat{\mathfrak{H}}$ is surjective shows that $\mathfrak{H}$ is surjective.

The proof of Theorem 3.2 also shows the following.

Theorem 3.3 Let $R, \mathcal{O}$, and $\Sigma$ be as in Theorem 3.1. Then the restriction of $J_{q}(\Gamma)_{\Sigma}$ to $W^{\prime}$ is a bounded isomorphism onto $\mathcal{D}(\Sigma)_{q}$.

Theorem 3.4 Let $R, \mathcal{O}$, and $\Sigma$ be as in Theorem 3.1. Let $q \in \Sigma$. If $h \in \mathcal{D}(\mathcal{O})$ then $\mathfrak{H} h=(h, 0)$, and if $h \in \mathcal{D}(\Sigma)_{q}$ then $\mathfrak{H} \mathfrak{O}(\Sigma, \mathcal{O}) h=(0,-h)$. 
Proof If $h \in \mathcal{D}(\mathcal{O})$, then since $\partial_{w} \mathscr{G}(w ; z, q)$ is holomorphic except for a simple pole of residue one at $w=z$, by the residue theorem $J_{q}(\Gamma)_{\mathcal{O}} h=h$ and $J_{q}(\Gamma)_{\Sigma} h=0$. The second claim is just Lemma 2.16.

We now prove Theorem 1.2.

Proof of Theorem 1.2 Let $h \in \mathcal{D}_{\text {harm }}(\mathcal{O})=\left(h_{1}, \ldots, h_{n}\right)$, and define

$$
\left(h_{\mathcal{O}}, h_{\Sigma}\right)=\mathfrak{H} h
$$

Theorem 3.4 yields

$$
\mathfrak{H}\left(-\mathfrak{O}(\Sigma, \mathcal{O}) h_{\Sigma}+h_{\mathcal{O}}\right)=\left(h_{\mathcal{O}}, h_{\Sigma}\right)=\mathfrak{H} h
$$

Thus by Theorem 3.2

$$
h=-\mathfrak{O}(\Sigma, \mathcal{O}) h_{\Sigma}+h_{\mathcal{O}}
$$

so $H=-H_{\Sigma}+H_{k}$ on $\Gamma_{k}$ for $k=1, \ldots, n$.

We need only show that the solution is unique. Given any other solution $\left(u_{\mathcal{O}}, u_{\Sigma}\right)$ we have that $-\mathfrak{O}(\Sigma, \mathcal{O})\left(u_{\Sigma}-h_{\Sigma}\right)+\left(u_{\mathcal{O}}-h_{\mathcal{O}}\right) \in \mathcal{D}_{\text {harm }}(\mathcal{O})$ has boundary values zero, so by uniqueness of the extension it is zero. Thus

$$
0=\mathfrak{H}\left(-\mathfrak{O}(\Sigma, \mathcal{O})\left(u_{\Sigma}-h_{\Sigma}\right)+\left(u_{\mathcal{O}}-h_{\mathcal{O}}\right)\right)=\left(u_{\mathcal{O}}-h_{\mathcal{O}}, u_{\Sigma}-h_{\Sigma}\right)
$$

which proves the claim.

\subsection{The Approximation Theorems}

In this section, we prove some approximation theorems for Dirichlet and Bergman spaces of nested Riemann surfaces, including Theorem 1.3 and Corollary 1.4.

Since the Dirichlet seminorm is not a norm, the meaning of density requires a clarification. Below, whenever we say that a linear subspace $Y$ of a Dirichlet space is dense in a Dirichlet space, the space $Y$ contains all constant functions. Thus, when we approximate in the Dirichlet seminorm, we are still free to adjust any "approximating" function by a constant without leaving $Y$.

Let

$$
\bar{P}_{\Omega_{k}}: \mathcal{D}_{\text {harm }}\left(\Omega_{k}\right) \rightarrow \overline{\mathcal{D}\left(\Omega_{k}\right)}
$$

denote orthogonal projection, where it is understood that for constants $c \bar{P}_{\Omega_{k}} c=c$. Let

$$
\oplus_{k} \bar{P}_{\Omega_{k}}: \oplus_{k} \mathcal{D}_{\text {harm }}\left(\Omega_{k}\right) \rightarrow \oplus_{k} \overline{\mathcal{D}\left(\Omega_{k}\right)}
$$

be the direct sum of these operators. 
Corollary 3.5 The image of $\oplus_{k} \mathfrak{G}\left(\Omega_{k, \epsilon}, \Omega_{k}\right) X_{\epsilon}$ under $\oplus_{k} \bar{P}_{\Omega_{k}}$ is dense in $W^{\prime}$.

Proof It is easily verified that the projection $\oplus_{k} \bar{P}_{\Omega_{k}}$ takes $W$ into $W^{\prime}$. Since it is a bounded surjective operator, the claim follows immediately from Theorem 2.10.

In the Corollary and its proof above, the reader should keep in mind that if $R$ has genus 0 , then the orthogonality conditions automatically hold. That is, $X_{\epsilon}=$ $\oplus_{k} \mathcal{D}\left(\Omega_{k, \epsilon}\right), W=\mathcal{D}_{\text {harm }}(\mathcal{O})$, and $W^{\prime}=\overline{\mathcal{D}(\mathcal{O})}$.

This leads to the following density theorem.

Theorem 3.6 The restrictions of functions in $\mathcal{D}\left(\operatorname{cl} \Sigma \cup \Omega_{1, \epsilon} \cup \cdots \cup \Omega_{n, \epsilon}\right)$ to $\Sigma$ are dense in $\mathcal{D}(\Sigma)$.

Proof Let $q \in \Sigma$. By Remark 2.9, the image of $W$ under $J_{q}(\Gamma)_{\Sigma}$ is equal to the image of $W^{\prime}$ under $J_{q}(\Gamma)_{\Sigma}$. By Theorem $3.3 J_{q}(\Gamma)$ is a bounded isomorphism from $W^{\prime}$ to $\mathcal{D}(\Sigma)_{q}$.

Thus by Corollary 3.5,

$$
J_{q}(\Gamma)_{\Sigma} \oplus_{k} \bar{P}_{\Omega_{k}}\left(\oplus \mathfrak{G}\left(\Omega_{k, \epsilon}, \Omega_{k}\right) X_{\epsilon}\right)
$$

is dense in $\mathcal{D}(\Sigma)_{q}$. Now Corollary 2.8 and Remark 2.9 yield that all of the functions in the set (3.1) have holomorphic extensions to cl $\Sigma \cup \Omega_{1, \epsilon} \cup \cdots \cup \Omega_{n, \epsilon}$. Since constant functions automatically have such extensions, this completes the proof.

Corollary 3.7 Let $R$ be a compact Riemann surface and $\Sigma \subset R$ be a Riemann surface such that the inclusion map is holomorphic and the boundary of $\Sigma$ consists of a finite number of pairwise disjoint quasicircles $\Gamma_{1}, \ldots, \Gamma_{n}$ in $R$.

Assume that there is an open set $\Sigma^{\prime} \subset R$ which contains $\Sigma$, and is bounded by quasicircles $\Gamma_{k}^{\prime}, k=1, \ldots, n$, which are isotopic in the closure of $\Sigma^{\prime} \backslash \Sigma$ to $\Gamma_{k}$ for $k=1, \ldots, n$, respectively. Then the set of restrictions of elements of $\mathcal{D}\left(\Sigma^{\prime}\right)$ to $\Sigma$ is dense in $\mathcal{D}(\Sigma)$.

Proof Consider the compact Riemann surface $R^{\prime}$ obtained from $\Sigma^{\prime}$ by sewing disks $\mathbb{D}$ to the quasicircles $\Gamma_{k}^{\prime}$ for $k=1, \ldots, n$ using fixed quasisymmetric parametrizations $\tau_{k}: \mathbb{S}^{1} \rightarrow \Gamma_{k}^{\prime}, k=1, \ldots, n$, say. It was shown in [9] that the topological space obtained from such a sewing has a unique complex structure compatible with that of $\Sigma^{\prime}$ and the sewn disks. Let $\Omega_{1}, \ldots, \Omega_{n}$ be the connected components of the complement of $\Sigma$ in $R^{\prime}$ containing $\Gamma_{1}^{\prime}, \ldots, \Gamma_{n}^{\prime}$, respectively. It follows from the hypotheses that each $\Omega_{k}$ is conformally equivalent to a disk bordered by $\Gamma_{k}$.

For each $k=1, \ldots, n$, fix a point $p_{k} \in \Omega_{k} \backslash \mathrm{cl} \Sigma^{\prime}$, and let $f_{k}: \mathbb{D} \rightarrow \Omega_{k}$ be conformal maps such that $f(0)=p_{k}$. We claim that for some $\epsilon>0, \Sigma^{\prime}$ is contained in cl $\Sigma \cup \Omega_{1, \epsilon} \cup \cdots \cup \Omega_{n, \epsilon}$. To see this, observe that the set $f_{k}^{-1}\left(\Gamma_{k}^{\prime}\right)$ is compact and does not contain 0 . Thus

$$
R_{k}=\inf _{p \in f_{k}^{-1}\left(\Gamma_{k}^{\prime}\right)}\{|p|\}>0
$$

Setting $r=\min \left\{R_{1}, \ldots, R_{n}\right\} / 2$ and $\epsilon=-\log r$ proves the claim. 
Applying Theorem 3.6 we obtain that $\mathcal{D}\left(\operatorname{cl} \Sigma \cup \Omega_{1, \epsilon} \cup \cdots \cup \Omega_{n, \epsilon}\right)$ is dense in $\mathcal{D}(\Sigma)$. Since $\mathcal{D}\left(\Sigma^{\prime}\right)$ contains the restrictions of elements of $\mathcal{D}\left(\operatorname{cl} \Sigma \cup \Omega_{1, \epsilon} \cup \cdots \cup \Omega_{n, \epsilon}\right)$ to $\Sigma^{\prime}$, this completes the proof.

We now address the case of one-forms.

Theorem 3.8 Let $R, \Sigma$, and $\Sigma^{\prime}$ be as in Corollary 3.7. Assume that $\Sigma^{\prime}$ (and hence $\Sigma$ ) is a bordered Riemann surface of genus $g$ and $n$ borders with $n \geq 1$. Then the set of restrictions of elements of $A\left(\Sigma^{\prime}\right)$ to $\Sigma$ is dense in $A(\Sigma)$.

Proof Let $R^{\prime}$ be the double of $\Sigma^{\prime}$. It is a surface of genus $2 g+n-1$, so the dimension of $A\left(R^{\prime}\right)$ is $2 g+n-1$. Let $a_{1}, \ldots, a_{2 g+n-1}$ denote a set of generators for the fundamental group of $\Sigma$. Given any $\alpha \in A(\Sigma)$, there is a $\beta \in A\left(R^{\prime}\right)$ with the same periods. Thus $\alpha-\beta$ is exact on $\Sigma$, with primitive $H$ say.

Thus by Corollary 3.7, for any $\epsilon>0$ there is an $h \in \mathcal{D}\left(\Sigma^{\prime}\right)$ such that

$$
\left\|H-\left.h\right|_{\Sigma}\right\|_{\mathcal{D}(\Sigma)}<\epsilon .
$$

Setting $\delta=\partial h+\left.\beta\right|_{\Sigma^{\prime}} \in A\left(\Sigma^{\prime}\right)$ we have that

$$
\left\|\alpha-\left.\delta\right|_{\Sigma}\right\|_{A(\Sigma)}=\left\|H-\left.h\right|_{\Sigma}\right\|_{\mathcal{D}(\Sigma)}<\epsilon
$$

This completes the proof.

The following example shows that the truth of Corollary 3.7 depends on the fact that every component of $R \backslash \Sigma$ contains a component of $R \backslash \Sigma^{\prime}$. Let $R=\overline{\mathbb{C}}$. Fix $r \in(0,1)$ and let $\Sigma=\{z: r<|z|<1\}$. For $0<r^{\prime}<r$ and $s^{\prime}>1$, Theorem 3.6 says that for $\Sigma^{\prime}=\left\{z: r^{\prime}<|z|<s^{\prime}\right\}, \mathcal{D}\left(\Sigma^{\prime}\right)$ is dense in $\mathcal{D}(\Sigma)$. However, setting instead $\Sigma^{\prime}=\mathbb{D}$, it is not true that $\mathcal{D}(\mathbb{D})$ is dense in $\mathcal{D}(\Sigma)$. To see this, fix $z \in \mathbb{C}$ such that $|z|>1$ and observe that the functional on $\mathcal{D}(\Sigma)$ given by

$$
\Lambda(h)=\left.J_{q}(\Gamma) h\right|_{z}
$$

is bounded, since point evaluation is bounded on the Dirichlet space of $\{z:|z|>$ $1\} \cup\{\infty\}$. This functional vanishes on $\mathcal{D}(\mathbb{D})$ but not on the entire space $\mathcal{D}(\Sigma)$. Thus $\mathcal{D}(\mathbb{D})$ is not dense in $\mathcal{D}(\Sigma)$.

Also, even removing a point from a component is not enough. Let $\Sigma^{\prime}=\mathbb{D} \backslash\{0\}$ and let $\Sigma$ be as above. Observe that any element of $\mathcal{D}(\mathbb{D} \backslash\{0\})$ extends to an element of $\mathcal{D}(\mathbb{D})$. Since $\mathcal{D}(\mathbb{D})$ is not dense in $\mathcal{D}(\Sigma)$ by the previous paragraph, the Theorem 3.6 does not extend to this case. On the other hand, the set of restrictions of holomorphic functions on $\mathbb{D} \backslash\{0\}$ to $\Sigma$ is dense in $\mathcal{D}(\Sigma)$.

We now prove Theorem 1.3 and Corollary 1.4.

Proof of Theorem 1.3 The set of restrictions of elements of $\mathcal{D}\left(\Sigma^{\prime \prime}\right)$ to $\Sigma$ contains the set of restrictions of elements of $\mathcal{D}\left(\Sigma^{\prime}\right)$ to $\Sigma$. Thus by Corollary 3.7, the set of restrictions of elements of $\mathcal{D}\left(\Sigma^{\prime \prime}\right)$ to $\Sigma$ are dense.

Similarly, by Theorem 3.8, the set of restrictions of elements of $A\left(\Sigma^{\prime \prime}\right)$ to $\Sigma$ is dense in $A(\Sigma)$. 
Proof of Corollary 1.4 Observe that $\Sigma^{\prime}$ can be viewed as a subset of its double $\Sigma^{D}$, and its boundary can be identified with $n$ analytic curves in the double. Thus the claim follows from Theorem 1.3 applied with $R=\Sigma^{D}$.

We indicate another possible approach to proving Theorem 3.8 (and therefore Theorem 1.3), using the result of Askaripour and Barron [2]. We assume that $\Gamma_{k}$ and $\Gamma_{k}^{\prime}$ are analytic curves for $k=1, \ldots, n$. Assume also that the universal cover of $R$ is the disk. Let $\pi: \mathbb{D} \rightarrow R$ be the covering map. Choose a collection of curves $\gamma_{j}$, $j=1, \ldots, g$ dissecting the compact surface $R$, where $g$ is the genus, to obtain a fundamental polygon $F$ in the disk $\mathbb{D}$. Choose the dissection such that every curve $\Gamma_{k}$ and $\Gamma_{k}^{\prime}$ is crossed by at least one of the dissecting curves. In that case, the sets $\pi^{-1}(\Sigma) \cap F$ and $\pi^{-1}\left(\Sigma^{\prime}\right) \cap F$ will be Carathéodory sets in the plane, and one can apply [2, Proposition 2.1] to obtain the result in the case of analytic curves.

One would need to show that such a dissection exists in general, which should not pose much difficulty. However, if one attempts this argument in the case of quasicircles, then establishing that the dissecting curves can be made to have the intersection property might be a delicate problem. On the other hand, if the dissecting curves are chosen not to intersect $\Gamma_{k}$ and $\Gamma_{k}^{\prime}$, the lifted sets $\pi^{-1}(\Sigma) \cap F$ and $\pi^{-1}\left(\Sigma^{\prime}\right) \cap F$ would not be Carathéodory sets, and one could not apply their result directly.

It should be noted that [2, Proposition 2.1] does not require analytic conditions on the boundary of $\Sigma$ and $\Sigma^{\prime}$, as we do in Theorem 3.8. Although we were able to remove the restrictions on the outer domain $\Sigma^{\prime \prime}$ to some extent in Theorem 1.3, we did not do so for $\Sigma$ itself. Thus their result suggests that the analytic conditions of Theorem 1.3 can be weakened.

\subsection{The Schiffer Comparison Operator for Open Surfaces}

Next we define a certain comparison operator, which generalizes an operator considered by Schiffer [4].

Let $R$ be a compact Riemann surface. Let $\Sigma$ and $\Sigma^{\prime}$ be Riemann surfaces such that $\Sigma \subseteq \Sigma^{\prime} \subseteq R$, and such that the inclusion maps from $\Sigma$ into $\Sigma^{\prime}$ and $\Sigma^{\prime}$ into $R$ are holomorphic. Define the restriction operator

$$
\begin{aligned}
R\left(\Sigma^{\prime}, \Sigma\right): A\left(\Sigma^{\prime}\right) & \rightarrow A(\Sigma) \\
\alpha & \left.\mapsto \alpha\right|_{\Sigma} .
\end{aligned}
$$

Assume that $\Sigma^{\prime}$ has a Green's function $\mathscr{G}_{\Sigma^{\prime}}$. We then define the Bergman kernel of $\Sigma^{\prime}$ to be

$$
K_{\Sigma^{\prime}}=-\frac{1}{\pi i} \partial_{z} \bar{\partial}_{w} \mathscr{G}_{\Sigma^{\prime}}(w, z)
$$

The Schiffer comparison operator is then defined to be

$$
S\left(\Sigma, \Sigma^{\prime}\right): A(\Sigma) \rightarrow A\left(\Sigma^{\prime}\right)
$$




$$
\alpha \mapsto \iint_{\Sigma} K_{\Sigma^{\prime}}(z, w) \wedge_{w} \alpha(w)
$$

We then have the following result, which strangely seems to have been missed by Schiffer, even in the planar case. By a hyperbolic metric, we mean a complete, constant negative curvature metric.

Theorem 3.9 Let $R$ be a compact Riemann surface, $\Sigma$ and $\Sigma^{\prime}$ be Riemann surfaces such that $\Sigma \subset \Sigma^{\prime} \subset R, \operatorname{cl} \Sigma \subset \Sigma^{\prime}$ and the inclusion maps from $\Sigma$ to $\Sigma^{\prime}$ and $\Sigma^{\prime}$ to $R$ are holomorphic. Assume that $\Sigma^{\prime}$ has a Green's function $\mathscr{G}_{\Sigma^{\prime}}(w, z)$, and that $\Sigma^{\prime}$ possesses a hyperbolic metric. Denoting the adjoint of $R\left(\Sigma^{\prime}, \Sigma\right)$ by $R\left(\Sigma^{\prime}, \Sigma\right)^{*}$ we have

$$
S\left(\Sigma, \Sigma^{\prime}\right)=R\left(\Sigma^{\prime}, \Sigma\right)^{*}
$$

Proof Let $\alpha \in A(\Sigma)$ and $\beta \in A\left(\Sigma^{\prime}\right)$. Then using the reproducing property of the Bergman kernel $K_{\Sigma^{\prime}}$ and assuming that we are allowed to interchange the order of integration we have

$$
\begin{aligned}
\left(S\left(\Sigma, \Sigma^{\prime}\right) \alpha, \beta\right)_{A\left(\Sigma^{\prime}\right)} & =\frac{i}{2} \iint_{\Sigma^{\prime}, z} \iint_{\Sigma, w} K_{\Sigma^{\prime}}(z, w) \wedge_{w} \alpha(w) \wedge_{z} \overline{\beta(z)} \\
& =-\frac{i}{2} \iint_{\Sigma, w} \iint_{\Sigma^{\prime}, z} \overline{K_{\Sigma^{\prime}}(w, z)} \wedge_{z} \overline{\beta(z)} \wedge_{w} \alpha(w) \\
& =-\frac{i}{2} \iint_{\Sigma} \overline{\beta(w)} \wedge_{w} \alpha(w) \\
& =\left(\alpha, R\left(\Sigma^{\prime}, \Sigma\right) \beta\right)_{A(\Sigma)} .
\end{aligned}
$$

To justify the change of the order of integration, let $\Sigma$ be compactly included in $\Sigma^{\prime}$ and $K_{n}$ be a sequence of compact subsets of $\Sigma$ that exhaust it (i.e., $K_{n} \rightarrow \Sigma$ ). Denote the $L^{p}$ norm over a set $U$ with respect to the hyperbolic metric on $\Sigma^{\prime}$ by $\|\cdot\|_{p, U}$ (see [10]). Note that the $L^{2}$ norm of a one-form (a one-differential in the terminology of [10]) with respect to the hyperbolic metric agrees with the $L^{2}$ norm used in this paper.

Now for fixed $w$ set

$$
c_{n}(w)=\left\|K_{\Sigma^{\prime}}(\cdot, w)\right\|_{1, K_{n}} .
$$

Note that $c_{n}(w)$ is a one-form on $\Sigma$ for every $n$ (of the form $a(w) d \bar{w}$ in local coordinates). Then

$$
\iint_{\Sigma, w} \iint_{K_{n}, z}\left|K_{\Sigma^{\prime}}(z, w) \wedge_{w} \alpha(w) \wedge_{z} \overline{\beta(z)}\right| \leq\|\beta\|_{\infty, K_{n}} \iint_{\Sigma, w}\left|c_{n}(w) \wedge_{w} \alpha(w)\right|
$$

so setting $C_{n}=\left\|c_{n}(\cdot)\right\|_{\infty, \Sigma}$ we have

$$
\iint_{\Sigma, w} \iint_{K_{n}, z}\left|K_{\Sigma^{\prime}}(z, w) \wedge_{w} \alpha(w) \wedge_{z} \overline{\beta(z)}\right| \leq C_{n}\|\alpha\|_{1, \Sigma}\|\beta\|_{\infty, K_{n}} .
$$


Now for the compact set $K_{n}$, a standard argument shows that there are constants $D_{n}$ such that $\|\beta\|_{\infty, K_{n}} \leq D_{n}\|\beta\|_{2, \Sigma}$ (see, e.g., [20, Lemma 2.1], which can be made global as in [10, Lemma 4.7]). Now the norm of the characteristic function on $\Sigma^{\prime}$ is finite so there is a constant $E$ such that $\|\alpha\|_{1, \Sigma} \leq E\|\alpha\|_{2, \Sigma} \leq E\|\alpha\|_{2, \Sigma^{\prime}}$. Thus

$$
\iint_{\Sigma, w} \iint_{K_{n}, z}\left|K_{\Sigma^{\prime}}(z, w) \wedge_{w} \alpha(w) \wedge_{z} \overline{\beta(z)}\right| \leq C_{n} D_{n} E\|\alpha\|_{2, \Sigma^{\prime}}\|\beta\|_{2, \Sigma}
$$

So Fubini-Tonelli's theorem applies and we have

$$
\begin{aligned}
& \iint_{K_{n}, z} \iint_{\Sigma, w} K_{\Sigma^{\prime}}(z, w) \wedge_{w} \alpha(w) \wedge_{z} \overline{\beta(z)} \\
& =-\iint_{\Sigma, w} \iint_{K_{n}, z} \overline{K_{\Sigma^{\prime}}(w, z)} \wedge_{z} \overline{\beta(z)} \wedge \alpha(w)
\end{aligned}
$$

Now since $\beta \chi_{K_{n}} \rightarrow \beta \chi_{\Sigma}$ in $L^{2}$ norm and the operator with kernel $K_{\Sigma}$ (or $\overline{K_{\Sigma}}$ ) was bounded on $L^{2}(\Sigma)$ then

$$
\iint_{K_{n}, z} \overline{K_{\Sigma^{\prime}}(w, z)} \wedge_{z} \overline{\beta(z)} \rightarrow \iint_{\Sigma} \overline{K_{\Sigma^{\prime}}(w, z)} \wedge_{z} \overline{\beta(z)}
$$

in the $L^{2}(\Sigma)$-norm. This, and (3.3) together with the fact that $\alpha \in L^{2}(\Sigma)$ (the dual of $L^{2}(\Sigma)$ ) yields that

$$
\begin{aligned}
& \iint_{\Sigma^{\prime}, z} \iint_{\Sigma, w} K_{\Sigma^{\prime}}(z, w) \wedge_{w} \alpha(w) \wedge_{z} \overline{\beta(z)} \\
& =-\iint_{\Sigma, w} \iint_{\Sigma^{\prime}, z} \overline{K_{\Sigma^{\prime}}(w, z)} \wedge_{z} \overline{\beta(z)} \wedge_{w} \alpha(w) .
\end{aligned}
$$

We now can prove the final result.

Proof of Theorem 1.5 Under the hypotheses, $\Sigma^{\prime}$ possesses a hyperbolic metric. It is obvious that $\operatorname{Ker}\left(R\left(\Sigma^{\prime}, \Sigma\right)\right)$ is zero. Thus,

$$
\mathrm{cl} \operatorname{Im}\left(S\left(\Sigma, \Sigma^{\prime}\right)\right)=\operatorname{Ker}\left(R\left(\Sigma^{\prime}, \Sigma\right)\right)^{\perp}=A\left(\Sigma^{\prime}\right)
$$

For the kernel, observe that by Corollary 3.7, the image of $R\left(\Sigma^{\prime}, \Sigma\right)$ is dense in $A(\Sigma)$. Thus

$$
\operatorname{Ker}\left(S\left(\Sigma, \Sigma^{\prime}\right)\right)=\left(\operatorname{cl} \operatorname{Im}\left(R\left(\Sigma^{\prime}, \Sigma\right)\right)\right)^{\perp}=\{0\} .
$$

This completes the proof. 
Remark 3.10 Note that operator $S\left(\Sigma, \Sigma^{\prime}\right)$ cannot have closed range, because that would imply that $R\left(\Sigma^{\prime}, \Sigma\right)$ has closed range. Since $R\left(\Sigma^{\prime}, \Sigma\right)$ is also injective, this would imply that it is surjective, which is clearly not the case.

Acknowledgements The authors are grateful to the referee, whose suggestions have improved the overall presentation of the paper.

Funding Open access funding provided by Uppsala University.

Open Access This article is licensed under a Creative Commons Attribution 4.0 International License, which permits use, sharing, adaptation, distribution and reproduction in any medium or format, as long as you give appropriate credit to the original author(s) and the source, provide a link to the Creative Commons licence, and indicate if changes were made. The images or other third party material in this article are included in the article's Creative Commons licence, unless indicated otherwise in a credit line to the material. If material is not included in the article's Creative Commons licence and your intended use is not permitted by statutory regulation or exceeds the permitted use, you will need to obtain permission directly from the copyright holder. To view a copy of this licence, visit http://creativecommons.org/licenses/by/4.0/.

\section{References}

1. Ahlfors, L.V., Sario, L.: Riemann Surfaces. Princeton Mathematical Series, vol. 26. Princeton University Press, Princeton, NJ (1960)

2. Askaripour, N., Barron, T.: On extension of holomorphic k-differentials on open Riemann surfaces. Houston J. Math. 40(4), 117-1126 (2014)

3. Bergman, S., Schiffer, M.: Kernel functions and conformal mapping. Compositio Math. 8, 205-249 (1951)

4. Courant, R.: Dirichlet's principle, conformal mapping, and minimal surfaces. With an appendix by M. Schiffer. Reprint of the original, p. 1977. Springer, New York, Heidelberg (1950)

5. Fornæss, J.E., Forstnerič, F., Fornæss Wold, E.: Holomorphic approximation: the legacy of Weierstrass, Runge, Oka-Weil, and Mergelyan. arXiv:1802.03924v3

6. Gakhov, F.D.: Boundary value problems. Translated from the Russian. Reprint of the: translation, p. 1990. Dover Publications Inc, New York (1966)

7. Gauthier, P.M., Sharifi, F.: Luzin-type harmonic approximation on subsets of non-compact Riemannian manifolds. J. Math. Anal. Appl. 474(2), 1132-1152 (2019)

8. Napalkov, V.V., Jr., Yulmukhametov, R.S.: On the Hilbert transform in the Bergman space. (Russian) Mat. Zametki 70 (2001), no. 1, 68-78; Translation in Math. Notes 70 (2001), no. 1-2, 61-70. Mathematica/Mathematische Lehrbücher, Band XXV. Vandenhoeck \& Ruprecht, Göttingen (1975)

9. Radnell, D., Schippers, E.: Quasisymmetric sewing in rigged Teichmüller space. Commun. Contemp. Math. 8(4), 481-534 (2006)

10. Radnell, D., Schippers, E., Staubach, W.: Quasiconformal maps of bordered Riemann surfaces with $L^{2}$ Beltrami differentials. J. Analyse Math. 132, 229-245 (2017)

11. Radnell, D., Schippers, E., Staubach, W.: A Model of the Teichmüller space of genus-zero bordered surfaces by period maps. Conform. Geom. Dyn. 23, 32-51 (2019)

12. Radnell, D., Schippers, E., Staubach, W.: Dirichlet spaces of domains bounded by quasicircles. Commun. Comtemp. Math. https://doi.org/10.1142/S0219199719500226

13. Radnell, D., Schippers, E., Shirazi, M., Staubach, W.: Schiffer operators and calculation of a determinant line in conformal field theory. Preprint

14. Royden, H.L.: Function theory on compact Riemann surfaces. J. Analyse Math. 18, $295-327$ (1967)

15. Rodin, Y.L.: Riemann boundary problem on Riemann surfaces. Mathematics and its Applications (Soviet Series), vol. 16. D. Reidel Publishing Co., Dordrecht (1988)

16. Schiffer, M.: The kernel function of an orthonormal system. Duke Math. J. 13, 529-540 (1946)

17. Schiffer, M., Spencer, D.: Functionals on finite Riemann surfaces. Princeton University Press, Princeton, N. J. (1954)

18. Schippers, E., Staubach, W.: Riemann boundary value problem on quasidisks, Faber isomorphism and Grunsky operator. Complex Anal. Oper. Theory 12(2), 325-354 (2018) 
19. Schippers, E., Staubach, W.: Transmission of harmonic functions through quasicircles on compact Riemann surfaces, To appear in Annales Academiae Scientiarum Fennicae Mathematica. arXiv: $1810.02147 \mathrm{v} 2$

20. Schippers, E., Staubach, W.: Plemelj-Sokhotski isomorphism for quasicircles in Riemann surfaces and the Schiffer operators. Math. Annalen. (2019). https://doi.org/10.1007/s00208-019-01922-4

21. Sharifi, F.: Uniform Approximation on Riemann Surfaces (2016). Electronic Thesis and Dissertation Repository. 3954. https://ir.lib.uwo.ca/etd/3954

22. Shen, Y.: Faber polynomials with applications to univalent functions with quasiconformal extensions. Sci. China Ser. A 52(10), 2121-2131 (2009)

Publisher's Note Springer Nature remains neutral with regard to jurisdictional claims in published maps and institutional affiliations. 